Collection SFN 11 (2010) 219-242

(C) Owned by the authors, published by EDP Sciences, 2010

DOI: $10.1051 / \mathrm{sfn} / 201011013$

\title{
Static and dynamic correlations in magnetic nanomaterials studied by small angle neutron scattering techniques
}

\author{
A. Wiedenmann \\ Institut Laue-Langevin, 38042 Grenoble Cedex, France \\ e-mail:wiedenmann@ill.eu
}

\begin{abstract}
The performance of new techniques developed for Small Angle Neutron Scattering (SANS) is illustrated by investigations on magnetic colloids. Polarised neutrons ("SANSPOL") are introduced as a special type of contrast variation for magnetic systems. Examples of diluted magnetic systems are reviewed where low magnetic contrasts had to be analysed beside strong nuclear contributions or vice versa. In Ferrofluids magnetic core-shell composite particles and magnetic aggregates could be precisely evaluated beside non-magnetic micelles and free surfactants of similar sizes. In more concentrated Ferrofluids an external magnetic field induces a pseudo-crystalline ordering which coexists with chain like arrangements of particles. Ordering and relaxation processes of magnetic moments in nanoparticles have been monitored by time-resolved SANS. Slow relaxation of magnetic particle moments onto equilibrium has been studied after switch off a static field. In stroboscopic experiments, time-frame data acquisition has been synchronized with a periodic external magnetic field. When a continuous neutron flux was used in conventional SANS, the shortest accessible time range was limited to about $3 \mathrm{~ms}$ resulting from the wavelength spread. A breakthrough of time resolution into the micro-second range was achieved with the pulsed frame overlap TISANE technique, which allows us to exploit a dynamical range similar to that of X-ray photon-correlation spectroscopy. The analysis of time-dependent SANS data as a function of frequency, field and temperature allowed i) to determine the underlying statistics describing the particle moment orientation, ii) to extract the effect of field-induced inter-particle correlations, iii) to monitor the slowing down of the dynamics of moment rotation with decreasing temperature, iv) to study the effect of freezing of the solvent on the dynamics of the particle moments, and v) to work out the possible relaxation mechanisms (Néel and Brownian).
\end{abstract}

\section{INTRODUCTION}

Small Angle Neutron Scattering (SANS) is perfectly adapted for studying density- and concentration fluctuations on a nanometer length scale between $0.5 \mathrm{~nm}$ and $300 \mathrm{~nm}$. This non-destructive technique allows different types of inhomogeneities to be identified in crystalline, amorphous as well as in liquid materials [1-3]. In addition, spatial fluctuations of the magnetisation can be monitored due to the interaction between neutron spin and magnetic moments. The magnetic behaviour of individual nanosized particles and the nature of their surfaces or interfaces can be precisely determined. In multiphase systems contrast variation techniques are needed in order to identify and to quantify the different components. When polarised neutrons (denoted as "SANSPOL") are used the relative scattering contrasts are strongly modified providing structural information unavailable in conventional SANS.

Dynamical investigations of nanosized inhomogeneities by means of SANS are usually limited to slow processes where during data acquisition time (typically of the order of a few minutes) the system remains in a quasi-steady state. The dynamical time is considerably reduced when such processes are

This is an Open Access article distributed under the terms of the Creative Commons Attribution-Noncommercial License 3.0, which permits unrestricted use, distribution, and reproduction in any noncommercial medium, provided the original work is properly cited. 
repeated in a reproducible pathway and data collection can be synchronised with the periodic process. We present new stroboscopic Small Angle Neutron Scattering (SANS) techniques which allow ordering and relaxation processes of magnetic moments in nanoparticle systems to be monitored. The aim of this paper is to illustrate the performance of these advanced SANS techniques which have been applied to various magnetic colloids. Magnetic single-domain nanoparticles in colloidal dispersions ("ferrofluids") spontaneously form dipolar chains. The presence of an external magnetic field not only can induce the growth and alignment of chains but also introduces lateral interactions between these chains, resulting in different types of local orderings and different dynamical behaviour.

This paper is organised as follows: In the second chapter we present the basic notations of SANS and the model functions that have been used to describe the SANS intensities for unpolarised and polarised neutrons. In the third chapter some results of SANSPOL investigations of the static nanostructures in diluted magnetic systems are presented where low magnetic contrasts had to be analysed beside strong nuclear contributions or vice versa. In more concentrated Ferrofluids an external magnetic field induces a pseudo-crystalline ordering which coexists with chain like arrangements of particles. In chapter 4 we show how time-dependent information can be obtained from continuous stroboscopic SANS and the pulsed beam TISANE technique and what are the limits. The relevant relaxation mechanisms of particle moments and the influence of the dipolar interactions are discussed followed by the concluding chapter 5 where the merits of these advanced SANS techniques are summarised.

\section{SMALL ANGLE NEUTRON SCATTERING TECHNIQUE}

Here we recall only basic notations of SANS the concept of which has been presented in various reviews e.g. [4]. From the "white" spectrum of the neutron source a small band of wavelengths $\Delta \lambda$ of the order of $\Delta \lambda / \lambda=10 \%$ is selected, collimated and directed onto the sample. Neutrons scattered around the primary beam are registered simultaneously in an area detector. Elastic scattering occurs at a scattering vector $Q$ when the neutron wavelength is unchanged by the scattering. This gives rise to a momentum transfer $Q=|Q|=4 \pi \sin \Theta / \lambda$ and hence to a phase shift at an atomic position $\mathbf{r}$ of $\exp (\mathrm{iQr})$. The total amplitude is obtained by summing up the scattering amplitudes of all atoms weighted by the phase shift at each atomic position. The scattered intensity $\mathrm{I}(\boldsymbol{Q})$ is the square of the total amplitude and measured in reciprocal space. By Fourier transform of $\mathrm{I}(\boldsymbol{Q})$ the correlation functions are obtained in real space giving access to size, composition and magnetization of inhomogeneities present in the material. In the SANS regime of low spatial resolution, the discrete atomic scattering amplitude of atoms can be replaced by a locally averaged scattering length density $\eta(\mathrm{r})$. A nuclear scattering length density is defined by $\eta_{\mathrm{N}}=\Sigma \mathrm{c}_{\mathrm{i}} \mathrm{b}_{\mathrm{i}} / \Omega_{\mathrm{i}}$, where $\mathrm{b}$ is the nuclear scattering length, $\mathrm{c}$ the concentration and $\Omega$ the atomic volume of the species i. Similarly, for the interaction between the neutron spin $S=1 / 2$ with an assembly of magnetic moments a magnetic scattering amplitude is defined by $\eta_{\mathbf{M}}=\left(\mathrm{e}^{2} \gamma / 2 \mathrm{mc}^{2}\right)$ $\Sigma c_{i} \mathbf{M}_{\mathbf{i}}^{\perp} / \Omega_{\mathbf{i}}$, where only the projection of the magnetic moment $\mathbf{M}_{\mathbf{i}}^{\perp}$ onto a plane perpendicular to the scattering vector $\mathbf{Q}$ contributes to the interaction. $\mathbf{M}_{\mathbf{i}}^{\perp}$ is given in units of Bohr magnetons $\mu_{\mathrm{B}}$ and $\left(\mathrm{e}^{2} \gamma / 2 \mathrm{mc}^{2}\right)=0.27^{*} 10^{-12} \mathrm{~cm}$. In two phase-systems (which we will consider throughout this paper) where particles are embedded in a homogeneous matrix the total scattering amplitude of the particle is called "form factor" and defined by

$$
\mathrm{F}(\mathrm{QR})=\int \mathrm{dr}^{3} \Delta \eta \exp \left(\mathrm{iQ} \mathbf{r}_{\mathbf{j}}\right)=\Delta \eta \mathrm{V}_{\mathrm{p}} \mathrm{f}(\mathrm{QR}),
$$

where $\mathrm{f}(\mathrm{QR})$ depends only on the shape of the particle and where the contrast $\Delta \eta$ is the difference between scattering length densities of particle and matrix, i.e. $\Delta \eta=\eta_{\mathrm{p}}-\eta_{\text {matrix }}$. Note that the magnetic contrast $\Delta \eta_{\boldsymbol{M}}$ and magnetic form factor $\boldsymbol{F}_{\boldsymbol{M}}^{\perp}(\boldsymbol{Q})$ are vectors and depend on the orientation of the moment with respect to $\mathbf{Q}$.

In poly-disperse multiphase systems when different types of particles $j$ of different shape $F_{j}(Q R)$ and size distributions $\mathrm{N}_{\mathrm{j}}(\mathrm{R})$ coexist all inhomogeneities will contribute to the scattering signal. If different 
particles of similar sizes are present their scattering contribution superimpose within the same Q-range. In concentrated systems interactions between particles can no longer be neglected. Such an assembly of interacting particles embedded in a homogenous matrix can be considered as a liquid, for which the particle positions are determined by the interaction potential $\mathrm{V}(\boldsymbol{r}) . \mathrm{V}(\boldsymbol{r})$ is related to the pair correlation function $g(r)$ which defines the probability to find a particle at a distance $r$ from a first particle at the origin. The Fourier transform of $\mathrm{g}(\mathrm{r})$ is measured in scattering experiments as the so called "structure factor” $S(Q)$ given by

$$
\mathrm{S}(\boldsymbol{Q})=1+\mathrm{N}_{\mathrm{p}} \int[\mathrm{g}(\boldsymbol{r})-1] \exp (\mathrm{i} \boldsymbol{Q} \boldsymbol{r}) \mathrm{d} \boldsymbol{r} .
$$

The scattered intensity therefore depends on the form factors $\mathrm{F}(\mathrm{Q}, \mathrm{R})$ and partial structure factors $\mathrm{S}_{i j}(\boldsymbol{Q})$ of all particles of type $i$ and $j$. When particles of size $R$ are assumed to be surrounded by particles of the same size the scattering is approximated by mono-disperse subsystems weighted by the size distribution according to $[5,6]$

$$
\mathrm{I}(\mathrm{Q})={ }_{\mathrm{j}} \Sigma \int \mathrm{N}_{\mathrm{pj}} F_{\mathrm{j}}{ }^{2}(\mathrm{QR}) \mathrm{N}_{\mathrm{j}}(\mathrm{R}) \mathrm{S}(\mathrm{Q}) \mathrm{dR} .
$$

$N_{j}(R) d R$ is the incremental volume fraction of particles of type $j$ in the radius interval between $R$ and $\mathrm{R}+\mathrm{dR}$ and $\mathrm{N}_{\mathrm{pj}}$ is the total particle number. For fully uncorrelated particles such as for a perfect gas $\mathrm{S}(\mathrm{Q})$ will be unity. In disordered condensed matter the scattering at large values of $\mathrm{Q}$ is determined mainly by the particle form-factor alone where $S(Q)=1$. For a repulsive potential such as excluded volume effects or electrostatic repulsion $\mathrm{S}(\mathrm{Q})$ decreases at low $\mathrm{Q}$ and oscillates with $\mathrm{Q}$ when the particles form a pseudo-periodic arrangement. Attractive interaction potentials will give rise to an increase of the intensity at low $\mathrm{Q}$ which might be confused with poly-dispersity or aggregates. A total structure factor $\mathrm{S}(\mathrm{Q})$ is experimentally derived by dividing the measured scattering intensities by the normalized intensities of very diluted samples where $S(Q)=1$ is assumed. Instead of the "very diluted" limit the intensities calculated from fits of the high $Q$ range can be used provided that the scattering contributions from form factors and structure factor can be reliably separated.

\subsection{SANS with polarised neutrons}

When neutron spins are aligned antiparallel, denoted by $(+)$, or parallel $(-)$ to a preferred orientation $\mathbf{z}$ $/ / \mathbf{H}$, four types of scattering processes are to be distinguished, two for conserving the neutron spin (snf) and two with reversal of the spin (sf) by the scattering process [7, 8]. When the spin state of the scattered neutrons is not analysed, the intensity collected in the detector contains both snf and sf contributions. We denote this technique as SANSPOL $[9,10]$.

For ferromagnetic single domain particles of saturation magnetization $\mathbf{M}_{\mathrm{s}}^{\mathrm{p}}$ embedded in a nonmagnetic matrix $[11,12]$ superparamagnetic behaviour is expected, when the orientation distribution of the magnetic moments in an effective magnetic field $\mathrm{H}_{\mathrm{eff}}$ follows the Langevin statistics according to

$$
\mathrm{L}(\mathrm{x})=\operatorname{coth}(\mathrm{x})-1 / \mathrm{x}
$$

where

$$
\mathrm{x}=\mathrm{M}\left(\mathrm{R}^{\prime}\right) \mathrm{H}_{\mathrm{eff}} / \mathrm{k}_{\mathrm{B}} \mathrm{T} .
$$

The total magnetic moment, $\mathrm{M}\left(\mathrm{R}^{\prime}\right)$, depends on the radius $R^{\prime}$ of the particles according to $\mathrm{M}\left(\mathrm{R}^{\prime}\right)=4$ $\pi \mathrm{R}^{\prime 3} \mathrm{~m}_{0} / 3 \Omega_{\text {at }}$ where $\mathrm{m}_{0}$ is the saturation value of the atomic magnetic moment. Using the formalism presented in $[12,13]$ SANSPOL cross sections $\mathrm{I}(-,+)(\mathbf{Q}, \alpha)$ for the case of non perfect alignment of magnetic moments have been derived to

$$
\begin{aligned}
\mathrm{I}(-,+)(\mathbf{Q}, \alpha)= & \left\{\left[\mathrm{F}_{\mathrm{M}}^{2} \mathrm{~L}^{2}(\mathrm{x}) \pm 2 \mathrm{~F}_{\mathrm{M}} \mathrm{F}_{\mathrm{N}} \mathrm{L}(\mathrm{x})\right] \sin ^{2} \alpha+\mathrm{F}_{\mathrm{N}}^{2}\right\} \mathrm{S}(\mathbf{Q}, \alpha) \\
& \left.+\mathrm{F}_{\mathrm{M}}^{2}\left\{2 \mathrm{~L}(\mathrm{x}) / \mathrm{x}-\sin ^{2} \alpha\left[\mathrm{L}^{2}(\mathrm{x})-1+3 \mathrm{~L}(\mathrm{x}) / \mathrm{x}\right)\right]\right\},
\end{aligned}
$$


where steric interaction effects were taken into account via phenomenological structure factors $S(\mathbf{Q}, \alpha)$. The intensities $\mathrm{I}^{+}(\mathbf{Q}, \alpha)$ and $\mathrm{I}^{-}(\mathbf{Q}, \alpha)$ are different for the two polarisation states for any angle $\alpha$ except for $\alpha=0$. The intensity difference between the two polarisation states is given by

$$
\mathrm{I}^{-}(\mathbf{Q}, \alpha)-\mathrm{I}^{+}(\mathbf{Q}, \alpha)=4 \mathrm{~F}_{\mathrm{N}} \mathrm{F}_{\mathrm{M}} \mathrm{L}(\mathrm{x}) \mathrm{S}(\mathbf{Q}, \alpha) \sin ^{2} \alpha .
$$

This nuclear magnetic cross-term is linear in the magnetic amplitude and hence makes it possible to determine the absolute value of the magnetic contrast with respect to the nuclear contrast, i.e. magnetic moment and compositions of particles and matrix. It allows further the structure factor $S(\mathbf{Q}, \alpha)$ for any direction $\alpha>0$ to be precisely determined beside the field variation of the magnetic moment according to the Langevin statistics. As will be shown later, eq. (5) helps to distinguish between inter-particle correlations and form-factors, which both may give rise to characteristic peaks in the SANS curves at low $\mathbf{Q}$.

Note that the average of the intensities for both neutron polarisations corresponds to the intensity of non-polarised neutrons given by

$$
\begin{aligned}
\mathrm{I}(\mathbf{Q}, \alpha)= & \left\{\left[\mathrm{F}_{\mathrm{M}}^{2} \mathrm{~L}^{2}(\mathrm{x}) \sin ^{2} \alpha+\mathrm{F}_{\mathrm{N}}^{2}\right\} \mathrm{S}(\mathbf{Q}, \alpha)\right. \\
& \left.+\mathrm{F}_{\mathrm{M}}^{2}\left\{2 \mathrm{~L}(\mathrm{x}) / \mathrm{x}-\sin ^{2} \alpha\left[\mathrm{L}^{2}(\mathrm{x})-1+3 \mathrm{~L}(\mathrm{x}) / \mathrm{x}\right)\right]\right\} .
\end{aligned}
$$

\subsection{SANSPOL combined with isotope contrast variation}

In poly-disperse multiphase systems the interpretation of complex SANS signals is generally not unique and requires special labelling techniques in order to identify and separate individual contributions. In some cases isotope composition of the matrix or particles can be adjusted in such a way that the contrast vanishes (contrast matching), i.e. the particle (or some part of it) will be "transparent" for neutrons. For SANSPOL labelling is due to the neutron-spin as an intrinsic physical variable which can be tuned [10]. This is illustrated in Fig. 1 for a magnetic composite particle, where the different grey scales represent the scattering length densities of a central magnetic core $\left(\eta_{1}\right)$, a nonmagnetic outer part $\left(\eta_{2}\right)$ and a nonmagnetic liquid as matrix $\eta_{\text {matrix }}$. Since $\eta_{1}^{(-,+)}=\eta_{1}^{\text {nuc }} \pm \eta_{1}^{\text {mag }}$ depend on the polarisation states $(+)$ or $(-)$ (black or tiled in Fig. 1) the scattering contrasts between core and shell as well as between core and matrix are different for both polarisation states. By switching the neutron polarisation from $(+)$ to $(-)$ two scattering curves $\mathrm{I}^{+}(\mathrm{Q})$ und $\mathrm{I}^{-}(\mathrm{Q})$ are measured alternatively on the same sample. Both curves then differ solely as a result of the different magnetic contrasts. In addition, the difference pattern $\mathrm{I}^{+}(\mathrm{Q})-\mathrm{I}^{-}(\mathrm{Q})$ is proportional to the product $\eta_{1}^{\text {mag }}{ }^{*} \eta_{1}^{\text {nuc }}$ of one and the same particle whereas nuclear and magnetic contrasts from different particles do not contribute to the cross-term. This allows nonmagnetic particles to be distinguished from magnetic particles even when they are of the same size. The "colour" of the surrounding matrix can be adjusted using the above mentioned contrast matching with different mixtures of isotopes in the solvent.

For all isotope mixtures both scattering curves $\mathrm{I}^{+}(\mathrm{Q})$ and $\mathrm{I}^{-}(\mathrm{Q})$, the difference $\mathrm{I}^{+}(\mathrm{Q})-\mathrm{I}^{-}(\mathrm{Q})$ and $\mathrm{F}_{\mathrm{N}}^{2}(\mathrm{Q})$ und $\mathrm{F}_{\mathrm{M}}^{2}(\mathrm{Q})$ as obtained from the averaged signal of eq. (6) can be adjusted simultaneously using the same structural model. By this combined contrast variation technique the number of possible structural models is considerably reduced. Further the consistence and accuracy of the constrained model parameters are hereby ensured [14].

\section{STATIC NANOSTRUCTURES IN MAGNETIC COLLOIDS}

"Ferrofluids" (denoted as FF) are magnetic liquids which consist of nanoscaled magnetic particles dispersed in a non-magnetic solvent [17]. Stable colloids are obtained when coagulation of the particles is prevented by electrostatic repulsion from surface charges or by coating the core with organic chain molecules acting as surfactants. Currently great effort is undertaken to prepare new 


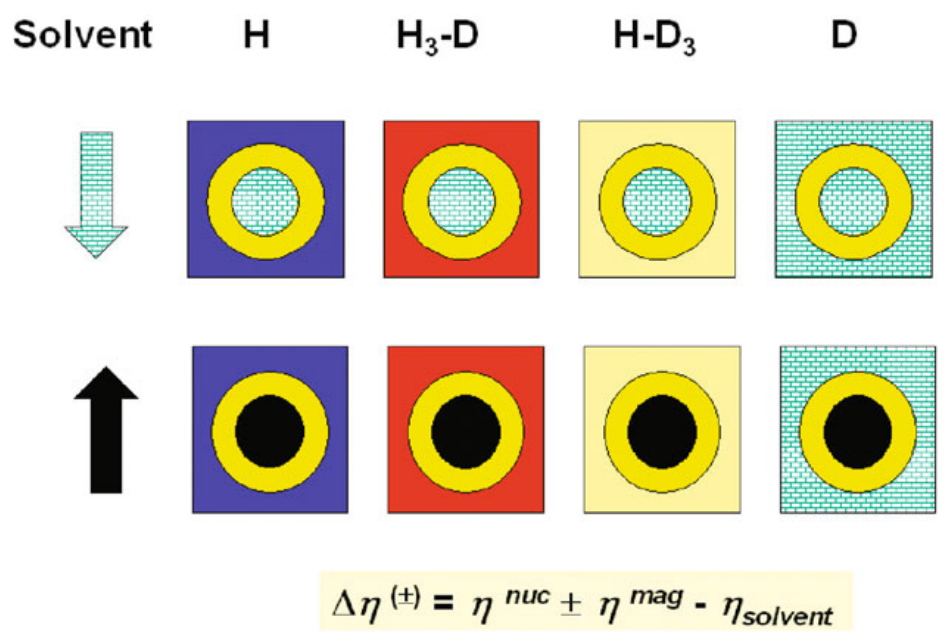

Figure 1. After [17] SANSPOL combined with isotope contrast matching as labelling technique illustrated by a grey scale representation of the scattering length densities $\eta$ of a composite particle in different surroundings: $\eta_{1}^{( \pm)}$ of the central magnetic core depends on the neutron polarisation. The contrast between the nonmagnetic shell and matrix is adjusted using different isotope mixtures of the solvent.

bio-compatible Ferrofluids for potential biomedical applications which necessitates a precise knowledge of the microstructural parameters.

\subsection{Experimental set-up}

SANS have been performed at the instruments V4 and D22 installed at the Helmholtz Zentrum, Berlin [15] and the Institut Laue Langevin, Grenoble respectively. Polarized neutrons are provided by a transmission polarizing super-mirror cavity [16]. The degree of polarisation is defined by $\mathrm{P}=$ $\left(\mathrm{n}^{+}-\mathrm{n}^{-}\right) /\left(\mathrm{n}^{+}+\mathrm{n}^{-}\right)$where $\mathrm{n}$ are the numbers of neutrons with spin antiparallel, $\mathrm{n}+$, and parallel to $\mathrm{H}, \mathrm{n}-$, respectively. The polarisation can be reversed from (+) to (-) in a spin flipper. Corrections for the actual values of polarisation and efficiency of the flipper have to be taken into account in the data analysis according to eq. (5). Static measurements have been performed by using an electromagnet with a strength of the magnetic field of up to $1.1 \mathrm{~T}$ applied horizontally and perpendicular to the incident beam.

\subsection{Core-shell structure of diluted Cobalt ferrofluids}

Colloidal solutions of ferromagnetic Cobalt can be prepared by coating the nano-sized particles with organic chain molecules $\mathrm{C}_{21}-\mathrm{H}_{39}-\mathrm{N}-\mathrm{O}_{3}$ [18]. In a first series [11] very diluted samples with a nominal concentration of 0.5 vol. \% of Cobalt have been prepared in mixtures of protonated and deuterated toluene, with a $\mathrm{C}_{7} \mathrm{D}_{8}$ content of $100 \%$ (denoted as AF2), $43 \%$ (AF4), $14 \%$ (AF3) and $0 \%$ (AF1). The 2-dimensional SANSPOL pattern for the sample AF4 shown in Fig. 2 are highly anisotropic with a dramatic change of the aspect ratios for the two polarization states. We emphasize the particularity that only for the polarization state $I^{-}$a maximum appears in the outer part of the patterns. The difference signal $\left(I^{-}-I^{+}\right)$of Fig. 2, where all background contributions are cancelled out, shows the angular dependence as expected from eq. (5) with negligible intensity along the direction of the magnetic field. Note that in the inner part the intensities are negative and positive in the outer part. SANSPOL intensities perpendicular to the applied field $I^{ \pm}(\mathbf{Q} \perp \mathbf{H})$ are compared for the different solvents in Fig. 3(a). Pronounced maxima occur only for $\left.I^{-}(\mathbf{Q} \perp \mathbf{H})\right)$ whereas $I^{+}(\mathbf{Q} \perp \mathbf{H})$ decreases always continuously 


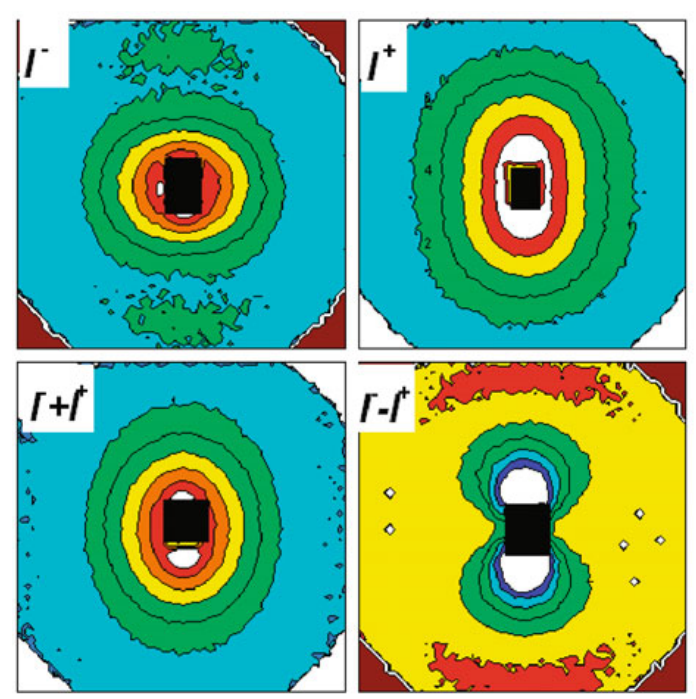

Figure 2. (After [11]) Co-ferrofluid AF4: SANSPOL patterns in for neutron spins antiparallel $\left(\mathrm{I}^{-}\right)$and parallel $\left(\mathrm{I}^{+}\right)$ to the horizontal field of strength $\mathrm{H}=1.1 \mathrm{~T}$. The arithmetic mean $\left[\left(\mathrm{I}^{-}\right)+\left(\mathrm{I}^{+}\right)\right] / 2$ corresponds to the 2D pattern of non-polarized neutrons. The difference $\left(\mathrm{I}^{-}\right)-\left(\mathrm{I}^{+}\right)$yields the interference term [equation (5)] which presents negative values in the center and positive values at high values of $\mathrm{Q}$ for vertical direction perpendicular to the magnetic field.

with increasing $Q$. The flipping ratio $\mathrm{FR}=I^{-}(\mathbf{Q} \perp \mathbf{H}) / I^{+}(\mathbf{Q} \perp \mathbf{H})$ is equal to unity at $Q=0.61 \mathrm{~nm}^{-1}$, FR $<1$ below and shows a maximum at $Q=0.8 \mathrm{~nm}^{-1}$ (Fig. 3). For Q larger than $1.35 \mathrm{~nm}^{-1}$, FR is again equal to unity, which proves that there is no magnetic contribution left. The "composite" is described by a shell model consisting of a sphere with an inner core radius $R^{\prime}$ surrounded by a concentric shell of thickness $\mathrm{D}$, i.e. $\mathrm{R}=\mathrm{R}^{\prime}+\mathrm{D}$. The form factor is given by

$$
F_{c-\text { shell }}(\mathrm{Q})=\left[\left(\Delta \eta_{1}-\Delta \eta_{2}\right) f_{s p h}\left(Q R^{\prime}\right)+\Delta \eta_{2} f_{s p h}(Q(R))\right] V_{p}
$$

with the shape function for spheres $f_{s p h}(x)=3[\sin (x)-x \cos (x)] / x^{3}$.

The scattering contrasts for the magnetic core $\Delta \eta_{1}^{(-,+)}=\eta_{1}^{n u c} \pm \eta_{1}^{\text {mag }}-\eta_{\text {matrix }}$ depends on the polarization while for non-magnetic shell $\Delta \eta_{2}=\eta_{2}^{n u c}-\eta_{\text {matrix }}$ is the same for both polarization states. The parameters $\eta_{1}^{\text {nuc }}, \eta_{1}^{\text {mag }}, \eta_{2}^{\text {nuc }}$ have been adjusted in a non-linear least square fitting routine using the constraints for the contrasts $\Delta \eta_{1}^{( \pm)}$and $\Delta \eta_{2}$ while $\eta_{\text {matrix }}$ of the solvent was known for the H/D ratio. The parameters $N_{p}, R_{0}$, D and $\sigma$ have been constrained to be identical for both polarization states. The solid lines in Fig. 3 represent the calculated intensities $I^{ \pm}(\mathbf{Q} \perp \mathbf{H})$ with the parameters presented in Fig. 3(d). The values of $\eta_{2}$ for the shell is of the same order of magnitude as calculated for densely packed surfactant molecules $\left(\eta_{2}=0.3310^{10} \mathrm{~cm}^{-2}\right)$ and do not depend significantly on the solvent composition. This supports strongly the conclusion that the shell of thickness of about $2.4 \mathrm{~nm}$ formed by organic surfactants is nearly impenetrable for the solvent in i.e. the Co-core is not in direct contact with the solvent. The values of $\eta_{1}(\mathrm{mag})=(3.9 \pm 0.2) 10^{10} \mathrm{~cm}^{-2}$ in AF2 and AF4 experimentally derived at $\mathrm{H}=1.1 \mathrm{~T}$ correspond closely to the magnetic moment of bulk Co.

\subsection{Multi-component ferrofluids}

A second series with nominal Co-concentrations as about 1 vol.\% have been prepared [18]. In a first step the SANSPOL difference pattern $(\mathrm{I}(+)-\mathrm{I}(-)$ was analyzed which only results from magnetic particles and where all nonmagnetic contributions are cancelled. Good agreement was obtained with 

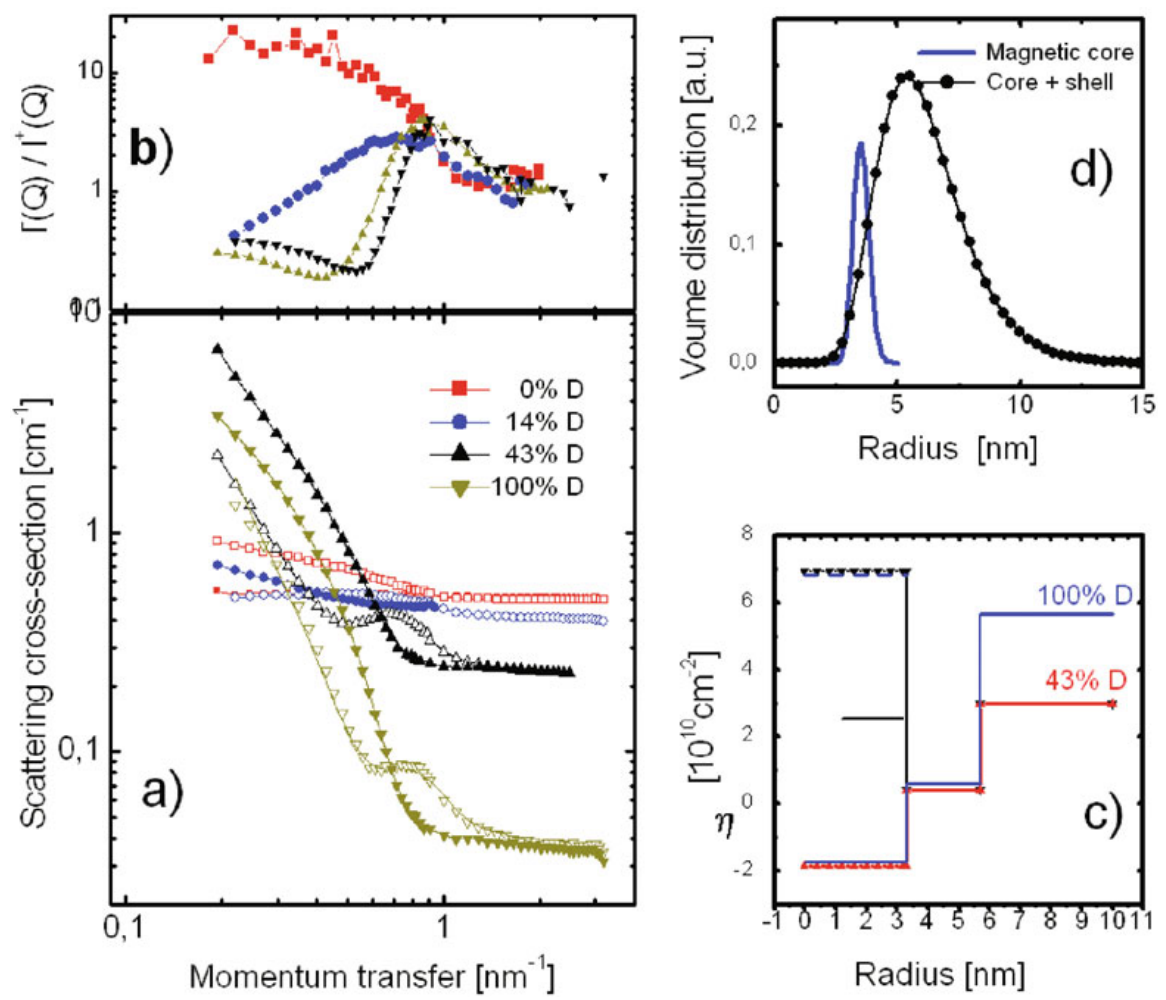

Figure 3. (After [11]) SANSPOL of Co-ferrofluids in different mixtures of $\mathrm{C}_{7} \mathrm{D}_{8}-\mathrm{C}_{7} \mathrm{H}_{8}$ : a) Intensities $\mathrm{I}^{+}(\mathrm{Q} \perp \mathrm{H})$ (solid symbols) and $\mathrm{I}^{-}(\mathrm{Q} \perp \mathrm{H})$ (open symbols). Solid lines: Fit according to a shell model using the parameters of Table 1. b) Flipping ratio $\mathrm{I}(-) / \mathrm{I}(+)$. c) Scattering length density profiles for two isotope mixtures of the solvents. d) volume distribution of a composite particle as a function of the radius.

parameters similar to those of the very diluted samples [14]). However, when these parameters where used to calculate the full curves $\mathrm{I}(+)$ and $(\mathrm{I}-)$ strong discrepancies occurred at higher values of $\mathrm{Q}$. This indicated the presence of an additional non-magnetic contribution resulting from excess surfactant molecules present in the solutions and which might form spherical micelles. Taking into account this additional contribution, all curves $I^{ \pm}(\mathbf{Q} \perp \mathbf{H}), F_{\mathrm{M}}^{2}$ and $F_{\mathrm{N}}^{2}$ could be adjusted simultaneously by the same core-shell model as described above with very similar values of the structural parameters. The fits revealed $1-5$ vol. \% of spherical non-magnetic particles with $\mathrm{R} \approx 1.8 \pm 0.4 \mathrm{~nm}$ which is very similar as the size of the magnetic particles. This example shows very instructively how SANSPOL allows different kind of magnetic and non-magnetic particles of very similar sizes to be identified.

\subsection{Superparamagnetic behaviour}

The variation of the magnetic contrast has been evaluated from the difference $\mathrm{I}^{-}(\mathrm{Q})-\mathrm{I}^{+}(\mathrm{Q})$ as a function of $\mathrm{H}$ and plotted in Fig. 4 [11]. The curves could be well fitted according to Eqs. (8b) and (9) by the product $\mathrm{F}_{\mathrm{N}} \mathrm{F}_{\mathrm{M}} \mathrm{L}(\mathrm{x})$ alone resulting in the particle size distribution shown in the inset of Fig. 4. This indicates that no significant inter-particle correlations are present or induced by the magnetic field, i.e. $S(Q \perp H)=1[11,19]$. The observed Langevin behaviour of the magnetic moments in the external magnetic field showed that the magnetic core is really composed by a single domain without significant anisotropy. 


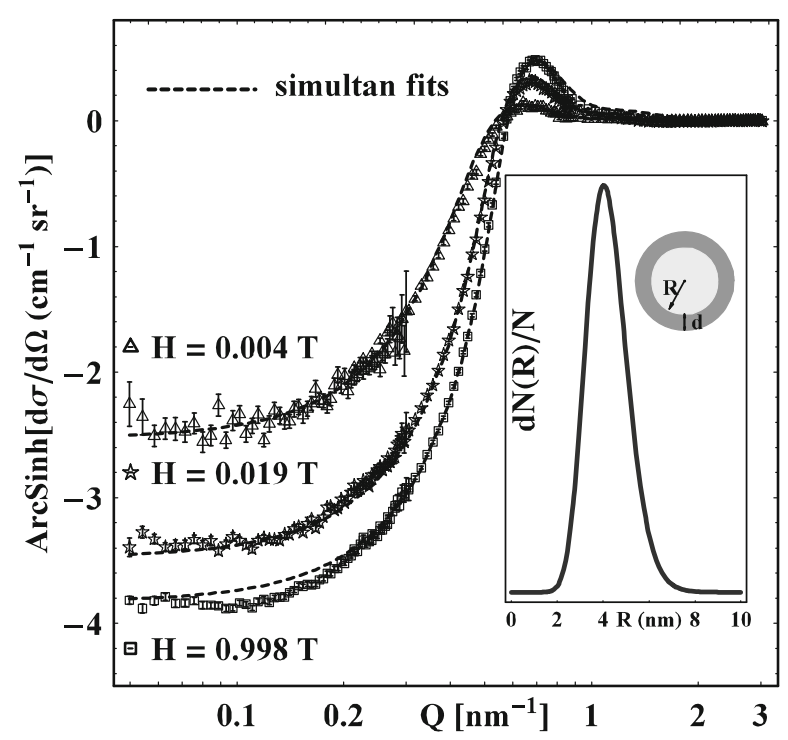

Figure 4. (After [19]) SANSPOL intensity difference of $\mathrm{D} 1$ at $\mathrm{H}=0.004 \mathrm{~T}(\Delta), 0.019 \mathrm{~T}$ (stars) and $0.998 \mathrm{~T}$ (squares.) The solid lines are simultaneous fits for non-interacting core-shell particles with the size distribution shown in the inset.

\subsection{Influence of core and surfactant materials}

A number of studies [20, 21] have been performed on aqueous Ferrofluids based on biocompatible Magnetite, $\mathrm{Fe}_{3} \mathrm{O}_{4}$, which are interesting for potential medical applications. The magnetite cores are charge stabilized which optionally can be coated by different surface active organic molecules forming bi-layers. The simultaneous model fitting revealed a major fraction of small particles with fully magnetised cores and a second fraction of particles with a radius of 2.5-3.5 times larger consisting of weakly magnetic aggregates containing Magnetite and surfactant materials. Apparently steric or electrostatic screening is not fully efficient in these magnetite based materials.

\subsection{Solvent dependent bonding of surfactants}

Magnetite nano-particles coated with Oleoylsarcosine (denoted as OLE) can be stabilized in water as well as in toluene as solvents. From a SANSPOL study it turned out that the shell thicknesses clearly depend on the solvents: In a non-polar solvent the layer thickness of $(1.9 \mathrm{~nm} \pm 0.1 \mathrm{~nm})$ is effectively characteristic for a monolayer just as found in other Cobalt Ferrofluids. However, in a polar solvent the shell is significantly larger $(2.3 \mathrm{~nm} \pm 0.1 \mathrm{~nm})$ which must correspond to a bi-layer. These results indicate that bonding of the surfactants to the magnetic core is mediated via the hydrophilic acid group and not via nitrogen atom [22].

\subsection{Oxydation stability}

The synthesis of iron particles from iron-carbonyl compounds results in particles with low polydispersity of which the particle size can be varied in a controlled way [23]. Ferrofluids of iron particles coated with oleic acid and dispersed in decalin have been kept under nitrogen atmosphere in order to prevent oxidation of the particles. SANSPOL-curves [24] for dilute samples with different isotope compositions of the solvent revealed the core composition $\mathrm{Fe}_{0.75} \mathrm{C}_{0.25}$. When exposed to air the SANSPOL curves 

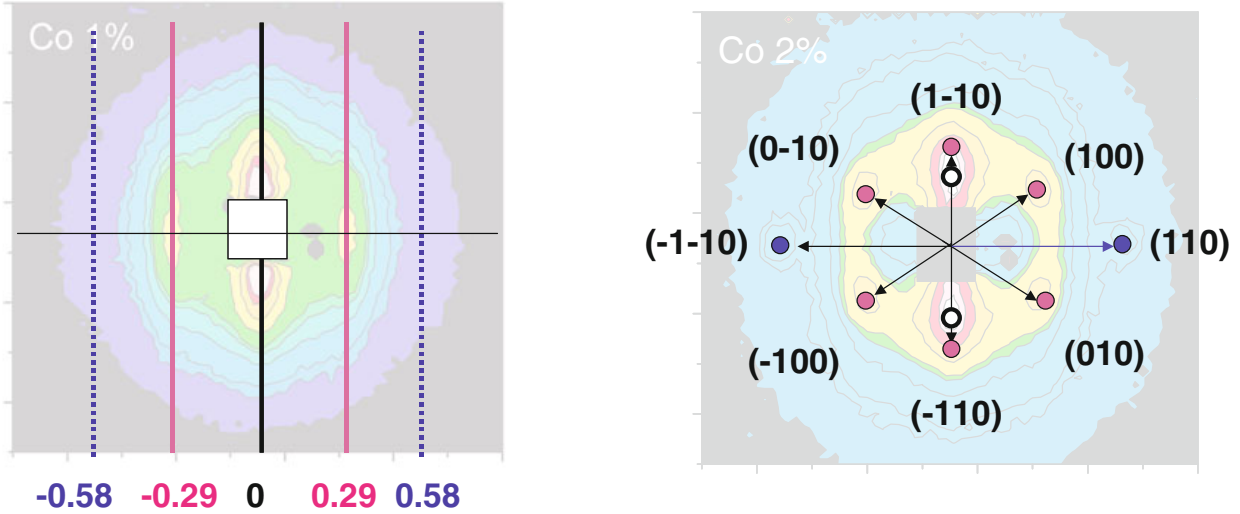

Figure 5. SANS-Iso-intensity pattern of 1 vol. \% Co-FF (left hand side) and 2 vol. \% Co FF (right hand side) in an horizontal field applied perpendicular to the neutron beam. While in the low concentrated sample only diffuse scattering appears in vertical planes at $Q_{\mathrm{x}}=0$ and $Q_{\mathrm{x}}= \pm 0.29 \mathrm{~nm}^{-1}$, the in-plane peaks observed in higher concentrated systems are assigned to a hexagonal symmetry with Millers indices corresponding to two textures.

could be well fitted with a two-shell-model consisting of the magnetic core surrounded by non-magnetic Fe-Oxide layer and the shell of surfactants.

\subsection{Particle correlations induced by magnetic fields}

Field induced correlations have been studied on a series of samples DS1-DS5 which have been prepared by dilution of a 6 vol. \% master sample [25]. The SANS pattern for non-polarised neutrons of samples DS1 (1 vol. \% Co) and DS2 (2 vol.\% Co) measured in a horizontal magnetic field of 1.1 $\mathrm{T}$ perpendicular to the incident beam are shown in Fig. 5. For Co concentrations above 1 vol. $\%$ pronounced peaks occurred at low Q, which disappeared in zero field. Six peaks are clearly distinguished at scattering vectors with constant lengths corresponding to $\mathrm{Q}_{1}=0.33 \mathrm{~nm}^{-1}$ and forming angles of $\pm 30^{\circ}$ and $\pm 90^{\circ}$ with the horizontal direction of the applied magnetic field. Two additional peaks appear at $\mathrm{Q}_{2}=0.57 \mathrm{~nm}^{1}$ in horizontal direction. In the vertical sector we find a strong and sharp peak at $\mathrm{Q}_{3}=0.24 \mathrm{~nm}^{-1}$. Fig. 5 illustrates how the peaks at $\mathrm{Q}_{1}$ are identified as reflections of a hexagonal lattice where $\mathrm{Q}_{1}$ correspond to the family $\{(100)\} \mathrm{Q}_{2}$ to the reflections $\{(110)\}$. The observed ratio of $\mathrm{Q}_{2} / \mathrm{Q}_{1}=1.73$ corresponds precisely to $\sqrt{ } 3$ as expected for hexagonal symmetry. The peaks observed in vertical direction at $Q_{3}=0.24 \mathrm{~nm}^{-1}$ were assigned to a second type of preferred orientation where the hexagonal planes are aligned parallel to the incident neutron beam i.e. with the [001] direction vertically. The observed 2D pattern is in fact a superposition of both textures where the (110) reflections at $\mathrm{Q}_{2}$ occur at the same position. Both textures are fully equivalent and should be equally probable. The structural arrangement perpendicular to the basal planes was studied in samples with 3 and 5 vol. \% Co (D3a and D5a) in a horizontal cryomagnet where the field up to $4 \mathrm{~T}$ was aligned parallel to the incoming neutrons. In this configuration $\mathbf{Q}$ is always perpendicular to the moment direction, i.e. $\sin ^{2} \alpha=1$ for all values of $\mathrm{Q}$, and fully isotropic patterns are expected from eq. (5-6). When the magnetic field is switched to $1 \mathrm{~T}$ a diffuse "Debye-Scherrer" ring is effectively observed at $\mathrm{Q}_{3}=0.08-0.1 \mathrm{~nm}^{-1}$ for $\mathrm{D} 3 \mathrm{a}$ ( $3 \%$ ) (see Fig. 6) and $\mathrm{Q}_{3}=0.21-0.23 \mathrm{~nm}^{-1}$ for the Co 5\% sample D5a. This diffuse peak is assigned to the (001) "powder" reflection showing the average distance $c_{\text {hex }}$ between the hexagonal layers. A quantitative evaluation of the SANSPOL data was performed using the structural model derived for the diluted Co-FF. The fitted curves plotted in left hand side of Fig. 6 as solid lines show the perfect agreement with the experimental data at high $\mathrm{Q}$. The structure factor $\mathrm{S}\left(\mathrm{Q}, \alpha=90^{\circ}\right)$ is now derived by dividing the experimental curves $\mathrm{I}(+)$ and $\mathrm{I}(-)$ by the corresponding functions calculated with the parameters as 

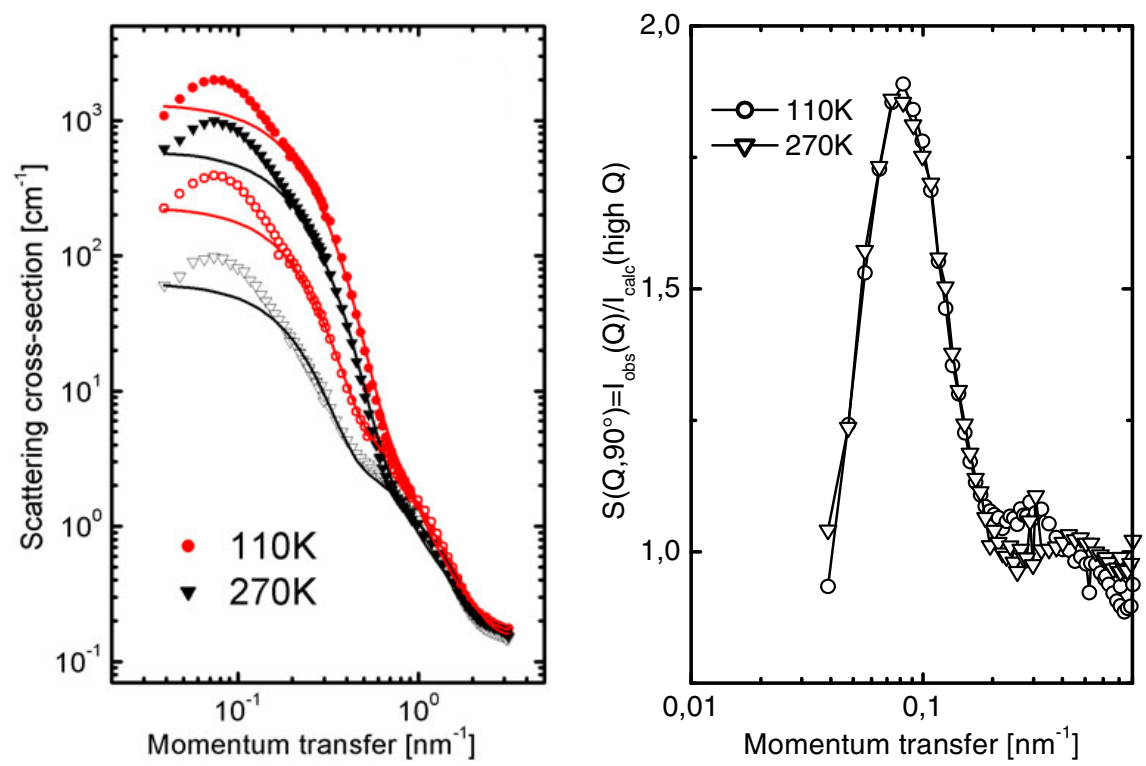

Figure 6. Left hand side.: Radial averaged SANSPOL-intensities $\mathrm{I}^{+}$and $\mathrm{I}^{-}$of sample D3a at $270 \mathrm{~K}$ and $110 \mathrm{~K}$ measured in a magnetic field of $0.3 \mathrm{~T}$ applied parallel to the neutron beam. The solid lines represent the high-Q fit corresponding to non-interacting core-shell particles. Right hand side.: Structure factors $\mathrm{S}\left(\mathrm{Q}, \alpha=90^{\circ}\right.$ ) (After [25]).

obtained from the model fits in the high $\mathrm{Q}$. The resulting $\mathrm{S}\left(\mathrm{Q}, \alpha=90^{\circ}\right)$, shown in right hand side of Fig. 6, is found to be almost unchanged between $270 \mathrm{~K}$ and $110 \mathrm{~K}$. The particle arrangement in both fully equivalent textures is represented in Fig. 7, together with the reciprocal lattice points observed in the detector plane. In the whole series of concentrated Co FF above 1 vol.\% Co inter-particle interactions are induced by an applied external magnetic field that gives rise to pseudo-crystalline ordering of Cobalt core-shell particles. The particles are arranged in a hexagonal symmetry with a particular preferential orientation of [110] along the magnetic field which gives rise to these two kinds of textures. The in-plane "lattice constants" $a_{\mathrm{hex}}=21.34 \mathrm{~nm}$ corresponding to the average distance of particles in the basal plane was found to be independent of the concentration while the "lattice constant" in c-direction depends on the concentration and varies between $23 \mathrm{~nm}$ and $31 \mathrm{~nm}$ in the DS2 to DS6 samples and between 70 and $25 \mathrm{~nm}$ for the series D3a and D5a, respectively. The ordering follows the direction of the applied field, i.e. the magnetic moments and the [110] directions are always aligned along the magnetic field. The size of these domains estimated from the width of the peaks corresponds to about $90 \mathrm{~nm}$, i.e. 4-5 "unit cells".

For the diluted sample with 1 vol. \% Co no such peaks were observed in an external field. Instead, diffuse residual intensities occur in planes perpendicular to the magnetic field at $Q_{\mathrm{x}}=0$ and $Q_{\mathrm{x}}= \pm 0.29 \mathrm{~nm}^{-1}$ and much less pronounced at $\mathrm{Q}_{\mathrm{x}}= \pm 0.58 \mathrm{~nm}^{-1}$ which strongly indicates the presence of chain-like aggregates of particles. The magnetic dipoles are arranged in the (attractive) headto tail configuration and which are aligned along the magnetic field. The reciprocal planes corresponding to this chaining are illustrated for 1 vol. \% Co FF in Fig. 5 (left hand side). Spontaneous chaining in zero field and coexistence of chains segments with hexagonal ordered domains has been observed when the magnetic field was increased [26]. Similar distorted hexagonal structures have been observed in Magnetite based ferrofluids by applying magnetic fields [43, 45].

The pseudo-crystalline lamellar hexagonal particle arrangement is unusual for magnetic colloids where hard-core repulsion competes with Van-der-Waals attraction and magnetic dipole-dipole interaction. The latter should give rise to a spontaneous arrangement of particles in chains or rings 

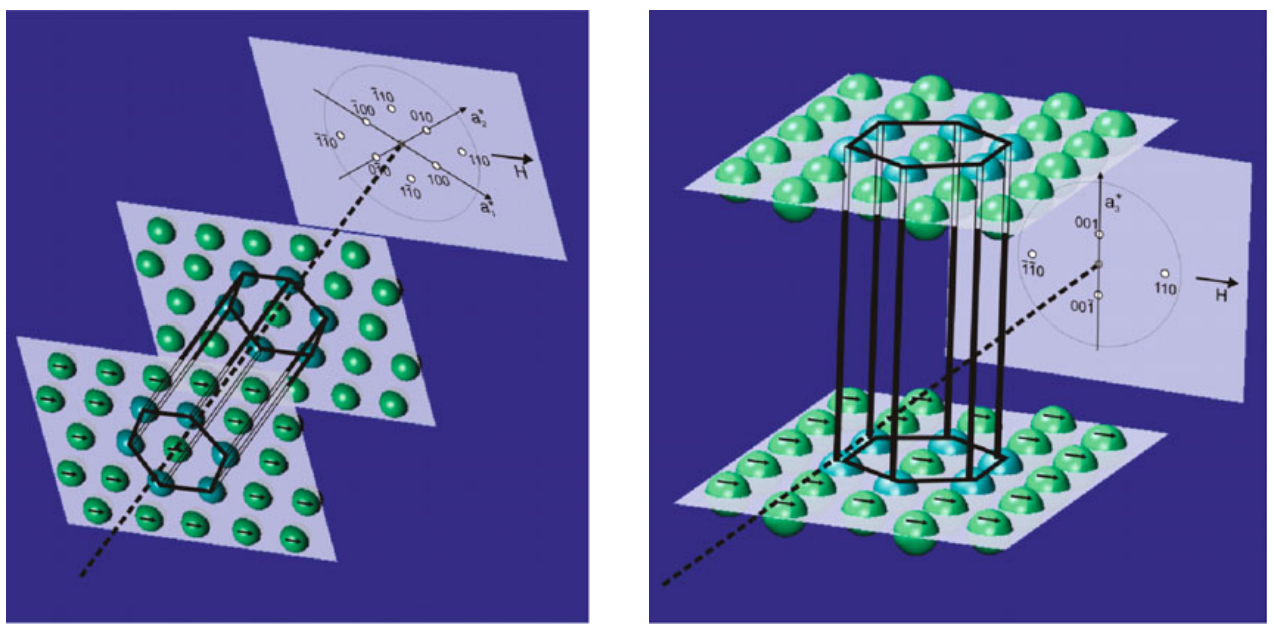

Figure 7. (after [25]) Schematic representation of particle arrangements in hexagonal symmetry in textures of type I (left hand side) and type II (right hand side) and the corresponding reciprocal lattice points observed in the detector plane. The horizontal magnetic field is perpendicular to the incident beam.

with magnetic moments parallel to each other [27]. Such spontaneous chaining in zero field has been predicted by of Monte-Carlo simulations [28] and recently observed by high-resolution cryogenic electron microscopy [23, 43]. In an external magnetic field these chains are expected to be aligned along the field direction that gives rise to anisotropic structure factors [29]. The possibility of pseudocrystalline particle arrangements is presently subject of theoretical treatments including Monte Carlo simulation and molecular dynamics studies [30-33].

\section{STROBOSCOPIC SANS TECHNIQUES}

In stroboscopic measurements a periodic perturbation (here a magnetic field) is applied to the sample. If a time dependent fluctuation of the scattering contrast is produced the response observed in the detector is a scattering pattern that changes as a function of time. For a reversible process the full cycle can be repeated until sufficiently high counting statistics is achieved on the detector, provided the detection is well synchronized and stable in time with the oscillating properties.

When a continuous neutron beam is used neutrons of a given wavelength hit the sample at a given phase of the AC magnetic field. The scattered neutrons arrive at the detector after a flight time $t_{\text {tof }}$ given by

$$
\mathrm{t}_{\mathrm{tof}}[\mathrm{ms}]=\lambda[\mathrm{nm}] \mathrm{L}_{\mathrm{SD}}[\mathrm{m}] 2.527778 \text {. }
$$

This leads to a delay of the response in the detector with respect to the magnetic field.

Figure 8 presents the distance versus time diagram of the continuous stroboscopic SANS technique. Neutrons of a fixed wavelength (solid lines) are scattered at the sample $(\mathrm{S})$ in the oscillation state defined by the frequency $v_{\mathrm{s}}$. Due to the flight time they are recorded in the detector (D) with some delay. When the detector acquisition frequency $v_{\mathrm{D}}$ is synchronised with $v_{\mathrm{s}}$, the time-resolved response (solid curve) is obtained with good statistics after a sufficient number of cycles. Due to the wavelength distribution of the incident neutrons $\Delta \lambda / \lambda$, typically of the order of $10 \%$, giving rise to the spread of $t_{\text {tof }}$ the response of faster (dashed pink lines) or slower neutrons (dotted blue lines) appears earlier (dashed pink curve) or later (dotted blue curve). The superposition of contributions at different wavelengths gives rise to a damping of the observed oscillations that increases with the frequency of the applied field. 


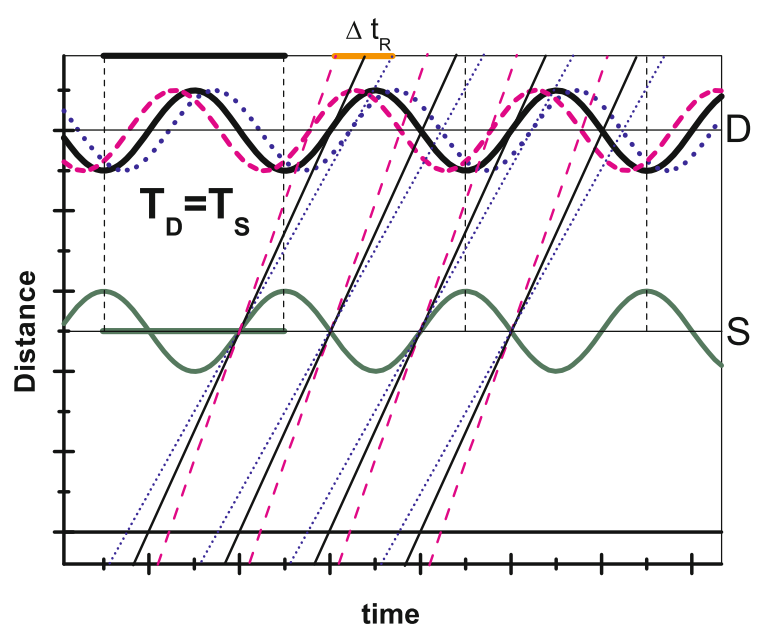

Figure 8. (after [42]) (Color online). Distance versus time diagram of the continuous stroboscopic SANS technique (see text).

Using the notation $\mathrm{T}_{\mathrm{s}}=1 / v_{\mathrm{s}}$ for the oscillation period of the magnetic field, the relative time spread $\varepsilon=\Delta \mathrm{t}_{\mathrm{R}} / \mathrm{T}_{\mathrm{s}}$ of the signal increases linearly with the frequency according to

$$
\varepsilon=\frac{\Delta \lambda}{\lambda} \cdot t_{t o f} \cdot v_{S}
$$

resulting in a smearing of the oscillations in the detector response (Fig. 8).

In conventional kinetics studies such as stopped-flow SANS experiments with typical periods of a few seconds the wavelength smearing can be neglected. This was also the case when we studied the decay of the local ordering in time slices of about $100 \mathrm{~ms}$ after switching off the magnetic field. However, when a sinusoidal magnetic field is applied the time resolution plays a crucial role. Hence for $v_{\mathrm{s}}=600 \mathrm{~Hz}$ the full period of the magnetic field is $\mathrm{T}_{\mathrm{s}}=1.66 \mathrm{~ms}$ which is already very close to the spread of neutron arrival times at $\mathrm{L}_{\mathrm{SD}}=4 \mathrm{~m}$ and $\lambda=0.608 \mathrm{~nm}$. The factors by which the original amplitudes of the response are reduced were evaluated to 0.93 at $100 \mathrm{~Hz}, 0.77$ at $200 \mathrm{~Hz}, 0.63 \mathrm{at} 300 \mathrm{~Hz}$ and to 0.11 at $600 \mathrm{~Hz}$. We note that for higher frequencies and/or longer distances the time resolution of the continuous stroboscopic technique is no longer sufficient to extract the oscillating response.

The response of the sample can be probed at much higher frequencies by using the pulsed TISANE technique [39] originally proposed by Gähler [40] (Fig. 9). A pulsed beam is produced by a chopper at a distance $\mathrm{L}_{1}$ from the sample. The chopper frequency $\nu_{\mathrm{E}}$ is locked-in with the oscillation frequency $v_{\mathrm{s}}$ of the magnetic field and the data acquisition frequency of the detector $v_{\mathrm{D}}$. All three frequencies are different and must satisfy the conditions

$$
v_{D}=v_{S}-v_{E} \quad \text { for } \quad v_{E} \cdot L_{1}=\left(v_{S}-v_{E}\right) \cdot L_{2} .
$$

Figure 9 shows that all neutrons from different chopper pulses which are elastically scattered by the sample in a given oscillation state are collected in the same time frame of the detector, irrespective of their wavelengths. Considerable gains in intensity and time resolution are obtained when a large frame overlap (i.e. long distances) and high repetition rates can be achieved. The time resolution at the detector is given by

$$
\Delta t_{R}^{2}=\left(\Delta t_{E} \cdot L_{2} / L_{1}\right)^{2}+\left[\Delta t_{S} \cdot\left(L_{1}+L_{2}\right) / L_{1}\right]^{2}+\left(\Delta t_{D}^{2}\right)
$$

which is dominated by the pulse length $\Delta \mathrm{t}_{\mathrm{E}}$. In the case of a double chopper system this value depends in turn on the frequency and the slit widths and can be varied typically between 50 and $500 \mu$ s. 


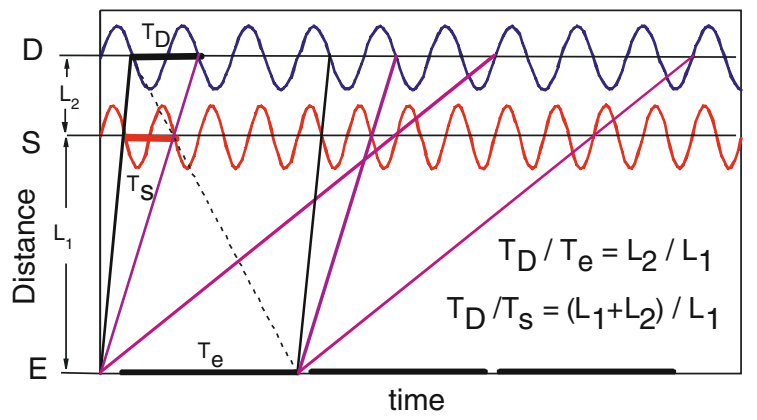

Figure 9. (after [39]). (Color online) Distance versus time diagram for the TISANE mode. A wide velocity band of neutrons emerges from successive chopper openings (E) causing frame overlap at the sample (S). Neutrons from different chopper pulses which probe the same oscillation state are observed at the same time channel at the detector (D), if eq. (10) is satisfied.

The contributions $\Delta \mathrm{t}_{\mathrm{S}} \approx \Delta \mathrm{t}_{\mathrm{D}} / 2 \approx 1.5 \mu \mathrm{s}$ are much shorter. Since time resolution $\Delta \mathrm{t}_{\mathrm{E}}$, sample cycle time $\mathrm{T}_{\mathrm{S}}$ and detection duty cycle $\mathrm{T}_{\mathrm{S}}$ scale inversely with the chopper frequency, the damping of the TISANE signal is independent of the oscillation frequency and much weaker than in the continuous SANS mode. Simulations of the TISANE signal revealed an optimum duty cycle for the chopper of about $15 \%$ [41] and showed that the conditions of eq. (10) have to be precisely kept during the whole measuring time.

\subsection{Experimental}

Two types of ferrofluids with nearly monodisperse nanoparticles have been investigated, namely concentrated Co-ferrofluid ("MFT3") $[26,39,44]$ with a cobalt core of radius $\mathrm{R}_{\mathrm{c}}=4.4 \mathrm{~nm}$ dispersed in a viscous synthetic hydrocarbon oil "Edwards L9" (monoalkylnaphthalene) and magnetite $\left(\mathrm{Fe}_{3} \mathrm{O}_{4}\right)$ particles of $R_{c}=8.5 \mathrm{~nm}$ dispersed in decalin [43,45] both stabilised by a surfactant layer of a thickness $\mathrm{D}$ of about $2 \mathrm{~nm}$. The maximal dipolar interaction energy $\mathrm{E}_{\mathrm{dd}}(\max )$ for particles with saturation magnetisation $\mathrm{M}_{\mathrm{sat}}$ in head-to-tail conformation and closest contact $\sigma=2\left(\mathrm{R}_{\mathrm{c}}+\mathrm{D}\right)$ is given by

$$
\mathrm{E}_{\mathrm{dd}}(\max )=-\mu_{0} \mathrm{M}_{\mathrm{sat}}^{2} \mathrm{~V}_{\mathrm{c}}^{2} /\left(2 \pi \sigma^{3}\right)
$$

and amounts to similar values for both ferrofluids. However, the viscosity of Co-FF system is by two orders of magnitudes higher than the $\mathrm{Fe}_{3} \mathrm{O}_{4}$-FF.

Stroboscopic experiments have been performed on the SANS instruments V4 at BENSC, Berlin, and D22 at ILL, Grenoble, using a continuous neutron beam with a wavelength band of $\Delta \lambda / \lambda=0.11$ at $\lambda=0.608 \mathrm{~nm}$. The area detector was placed at a distance of $\mathrm{L}_{\mathrm{SD}}=4 \mathrm{~m}$ from the sample. For the study of the long term relaxation [44] the ferrofluid samples have been placed in a homogeneous horizontal magnetic field B applied perpendicular to the incoming neutrons. The field could be switched off from $0.5 \mathrm{~T}$ to the remanent field of $0.005 \mathrm{~T}$ within less than $100 \mathrm{~ms}$. Time-frame histogram SANS measurements have been performed in time-slices of $500 \mathrm{~ms}$ during a total time of $15 \mathrm{~s}$ after switching off the magnetic field. For raising the field and stabilize it at $0.5 \mathrm{~T}$ a waiting time of $5 \mathrm{~s}$ was intercalated. Sufficient counting statistics were obtained after 200-400 cycles. Due to the low ramp-up rate of the magnetic field, kinetics of ordering process could not be observed by this stroboscopic technique. Using polarised neutrons without analysis of the polarisation of scattered neutrons (SANSPOL) [46] the scattering intensities $\mathrm{I}(+)$ and $\mathrm{I}(-)$ have been measured with incident neutron spin parallel $(-)$ and antiparallel $(+)$ to the magnetic field. The difference in intensities allowed the nuclear magnetic interference term to be precisely evaluated. 
For the study of the re-ordering process a different set-up has been used. A magnetic field generating coil was built around a ferrite core and equipped with a high-stability frequency generator and power supply. Periodic sine-wave modulations of the horizontal magnetic field up to amplitudes of $\mathrm{B}_{0}=40 \mathrm{mT}$ and frequencies between $v_{\mathrm{s}}=50 \mathrm{~Hz}$ and $500 \mathrm{~Hz}$ have been applied to the sample placed in the homogenous part of the magnetic field. Optionally, a static field up to $B_{\text {st }}=20 \mathrm{mT}$ could be superimposed. The reversibility of the reordering process in the dynamic mode has been checked by comparing the stroboscopic SANS results with those measured in the same coil device powered by a DC current that produces a static magnetic field of the same amplitude. Data acquisition was triggered by the frequency generator at zero AC voltage producing histograms of $128 \times 128$ pixels of $0.5 \times 0.5 \mathrm{~cm}^{2}$ (V4) and $0.8 \times 0.8 \mathrm{~cm}^{2}(\mathrm{D} 22)$ for position and $\mathrm{n}=100$ time channels of width $\Delta \mathrm{t}=\left(\mathrm{n} v_{\mathrm{s}}\right)^{-1}$.

The TISANE principle has been first realized at the time-of-flight instrument NEAT at BENSC, using a fast counter rotating double chopper with a time resolution of 3.5\%. Chopper frequencies could be varied up to $v_{\mathrm{E}}=666 \mathrm{~Hz}$ at $\mathrm{L}_{1}=13 \mathrm{~m}$, which according to eq. (10) allowed a maximum frequency of the AC field of $v_{\mathrm{s}}=2800 \mathrm{~Hz}$. Using the "white beam" with $0.2 \mathrm{~nm}<\lambda<2 \mathrm{~nm}$ a $Q$-range between 0.1 and $4 \mathrm{~nm}^{-1}$ was covered. The $\mathrm{Q}$ resolution was relaxed to the order of $\Delta \mathrm{Q} / \mathrm{Q} \approx 0.35$.

In all cases intensities have been corrected pixel by pixel for background, transmission and detector efficiency and normalised to the scattering of a water standard using the software BERSANS. The $\mathrm{Q}$ range $0.2 \mathrm{~nm}^{-1}<\mathrm{Q}<1.2 \mathrm{~nm}^{-1}$ and resolution of the order of $\Delta \mathrm{Q} / \mathrm{Q}<0.3$ allowed us to resolve properly the characteristic correlation peaks of the local hexagonal ordering that appear for the CoFF samples at $\mathrm{Q}_{1}=0.4 \mathrm{~nm}^{-1}$ and $\mathrm{Q}_{2}=0.3 \mathrm{~nm}^{-1}[8,9]$ and for $\mathrm{Fe}_{3} \mathrm{O}_{4}$ at $\mathrm{Q}_{1}=0.36 \mathrm{~nm}^{-1}$ and $\mathrm{Q}_{2}=$ $0.34 \mathrm{~nm}^{-1}$ [45]. In order to analyse the full time dependence, intensities have been integrated in angle sectors over a width of $20^{\circ}$ at angles $\alpha=0^{\circ}, 30^{\circ}, 60^{\circ}$, and $90^{\circ}$. Data analysis by simultaneous fits was performed using the software SASfit.

\subsection{Dynamical scattering cross-sections}

In the case where the magnetic field is switched off within a time much shorter than the stroboscopic time slices the time dependent intensity describes the decay of the correlations acquired in equilibrium state at the static magnetic field B.

Applying an oscillating magnetic field described by

$$
\mathrm{B}(\mathrm{t})=\mathrm{B}_{0} \sin \left(2 \pi v_{\mathrm{s}}\left(t+t_{\mathrm{tof}}\right)+\varphi\right)+\mathrm{B}_{\mathrm{st}}
$$

where $\mathrm{B}_{0}$ and $\varphi$ are the amplitude and arbitrary phase of the oscillating field and $\mathrm{B}_{\text {st }}$ is the superimposed static field, a time dependence enters in eq. (4)-(6) via the argument $x$ of the Langevin function. The term in braces has to be convoluted with $\mathrm{p}(\lambda, \Delta \lambda)$ describing the triangular wavelength distribution to account for the smearing of the oscillations. The time dependent intensity is given by

$$
\begin{aligned}
\mathrm{I}(\mathbf{Q}, \alpha, \mathrm{B}, \mathrm{t})= & \mathrm{f}_{\mathrm{e}} \int\left\{\left[\mathrm{F}_{\mathrm{M}}^{2} \mathrm{~L}^{2}(\mathrm{x}) \sin ^{2} \alpha+\mathrm{F}_{\mathrm{N}}^{2}\right\} \mathrm{S}(\mathbf{Q}, \alpha)+\mathrm{F}_{\mathrm{M}}^{2}\{2 \mathrm{~L}(\mathrm{x}) / \mathrm{x}\right. \\
& \left.\left.-\sin ^{2} \alpha\left[\mathrm{L}^{2}(\mathrm{x})-1+3 \mathrm{~L}(\mathrm{x}) / \mathrm{x}\right)\right]\right\} \mathrm{p}(\lambda, \Delta \lambda) \mathrm{d} \lambda+(1-\mathrm{fe}) \mathrm{U}\left(\mathrm{Q}, \alpha, v_{\mathrm{s}}\right) .
\end{aligned}
$$

The factor $f_{e}$ gives the fraction of superparamagnetic moments that can follow the oscillating field, whereas $\left(1-\mathrm{f}_{\mathrm{e}}\right)$ is the fraction of moments that cannot follow and leads to a time-independent scattering contribution $\left(1-\mathrm{f}_{\mathrm{e}}\right) \mathrm{U}\left(\mathrm{Q}, \alpha, v_{\mathrm{s}}\right)$.

The important question is whether the effective dynamical structure factor $S(Q, \alpha, t)$ in eq. (14) will depend on the applied magnetic field. If not, the intensity will depend on the magnetic field only via the Langevin parameter $\mathrm{x}$ according to eq. (4). In the direction along the magnetic field, for $\alpha=0$, the integral term of eq. (14) simplifies to $F_{N}^{2} S(Q, \alpha=0, t)+F_{M}^{2} 2 L(x) / x$ which yields a maximum at $B=0$, since $2 L(x) / x=2 / 3$ while for finite magnetic fields this term is smaller than $2 / 3$ and vanishes for full alignment of the particle moments. For all other directions, additional magnetic terms add 


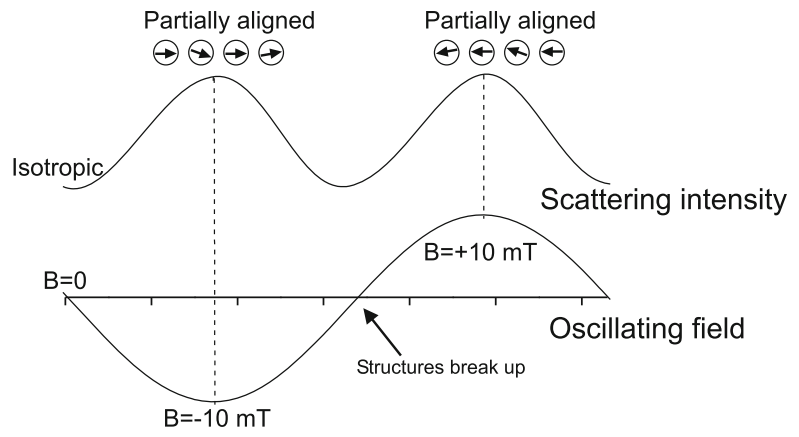

Figure 10. After [42] Schematic illustration of the scattering intensity of a ferrofluid as a function of the applied oscillating magnetic field. At $\mathrm{B}=0$, the scattering pattern is nearly isotropic (assuming immediate breakup of the structures), while at $\mathrm{B}= \pm 10 \mathrm{mT}$ the magnetic moments become aligned, leading to anisotropic scattering patterns.

up in such a way that at $B=0$ the intensity $I(\alpha)$ will always be lower than in the maximum field. This means that the intensities are expected to oscillate in an opposite way as a function of time. Conversely, in-phase oscillating intensities for all angles can only be observed when the structure factor $\mathrm{S}(\mathrm{Q}, \alpha, \mathrm{t})$ itself depends on the magnetic field. It that case, the term $\mathrm{F}_{\mathrm{N}}^{2} \mathrm{~S}(\mathrm{Q}, \alpha=0, \mathrm{t})$ increases with $\mathrm{B}$ leading to an intensity maximum at $\mathrm{B}_{\max }$ and minimum at $\mathrm{B}=0$. Note that only in the case where $\mathrm{F}_{\mathrm{N}}^{2} \mathrm{~S}(\mathrm{Q}, \alpha, \mathrm{t})>(2 / 3) \mathrm{F}_{\mathrm{M}}^{2}$ is the dominant term the time-dependent structure factor can be extracted reliably from the oscillating intensities. This condition is clearly fulfilled in the $\mathrm{Fe}_{3} \mathrm{O}_{4}$ - $\mathrm{FF}$ samples for which $\Delta \eta($ nuc $) / \eta \Delta$ (mag) $\sim 5$. This implies that for all angles the scattering intensity follows the oscillating field as is depicted in Fig. 10. For Co-FF, where $\Delta \eta($ nuc $) / \eta \Delta(\mathrm{mag})=0.28$, anti-phase oscillations are expected.

\subsection{Relaxation of magnetic correlations towards equilibrium}

The scattering patterns measured at $\mathrm{H}=0.5 \mathrm{~T}$ and $\mathrm{H}=0$, respectively, turned out to be identical when measured in the stroboscopic cycling or in the static mode which demonstrated that the orderingdisordering process was really reversible.

The scattering intensities after averaging the 2D patterns at angles $\alpha=90^{\circ}$ and $\alpha=30^{\circ}$ over a width of $\Delta \alpha=20^{\circ}$ have been analysed as a function of the momentum transfer $\mathrm{Q}$. The peaks which define the local hexagonal order clearly decay with time. In the final relaxation state the sum intensities show a $Q^{-1}$ dependence at low $\mathrm{Q}$ which is a characteristic feature of cylindrical objects. This behavior is assigned to short segments of dipolar chains. The difference pattern exhibits a similar $\mathrm{Q}^{-1}$ behavior in particular in the vertical sector $\left(\alpha=90^{\circ}\right)$. In the $30^{\circ}$ sector a $\mathrm{Q}^{-1}$ contribution at low $\mathrm{Q}$ first increases during initial time steps and decreases later. This shows that the hexagonal order gradually transforms to chain segments. Even after full relaxation, particles are still partly arranged in segments of chains in headto-tail conformation of the dipoles with some alignment along the residual remanent field. Since the decay of the peak intensities was observed as well in the sum as in the difference patterns we conclude that nuclear inter-particle correlations disappear simultaneously with the magnetic correlations. The SANSPOL difference intensities averaged over boxes in the angle sectors at $\alpha=90^{\circ}$ and $30^{\circ}$ are plotted in Fig. 11 as a function of time. All curves are well fitted by a single exponential decay according to $\mathrm{I}(\mathrm{t})=\mathrm{y}_{0}+\mathrm{A} \exp (-\mathrm{t} / \tau)$ where $\tau$ represents a characteristic relaxation time.

While in the $30^{\circ}$ sector the relaxation time $\tau\left(\mathrm{Q}_{1}\right)=3.4 \mathrm{~s}$ is maximum at the in-plane correlation peak, $\tau\left(\mathrm{Q}_{3}\right)=2.4 \mathrm{~s}$, at the inter-plane correlation is considerably shorter. A continuous increase of $\tau$ was found in the vertical sector where the longest relaxation times of $6 \mathrm{~s}$ where found at $0.1 \mathrm{~nm}^{-1}$ which corresponds to the longest segments of chain composed by about 4-5 particles. 


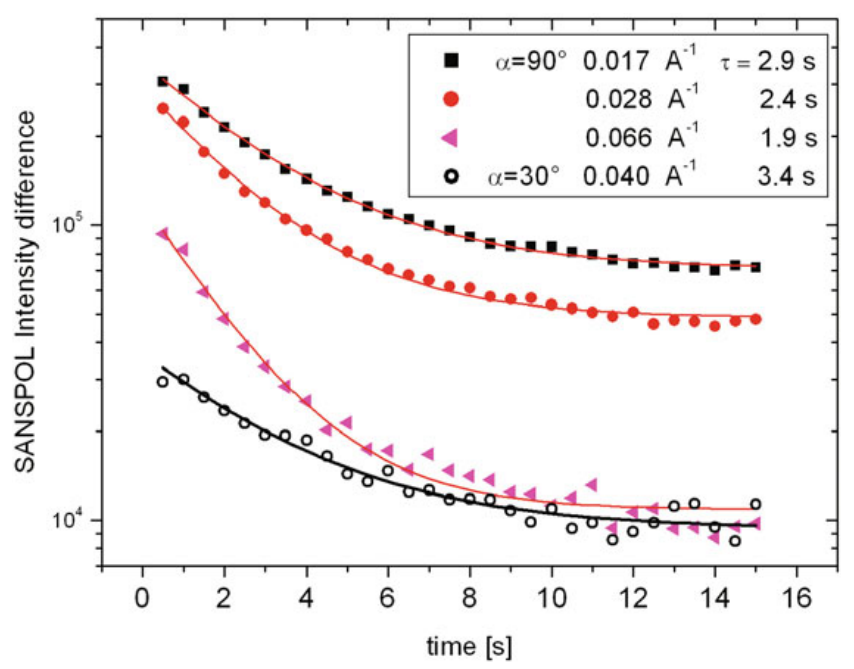

Figure 11. (after [44]) Time decay of the SANSPOL intensity differences in sectors perpendicular to $\mathrm{H}\left(\alpha=90^{\circ}\right)$ at different values of $\mathrm{Q}$ (solid symbols) and at $\mathrm{Q}_{1}$ in the sector $30^{\circ}$ (open cercles).The solid lines correspond to fits of an exponential decay with the Q dependant time constants $\tau$.

\subsection{Response to an oscillating field in continuous stroboscopic SANS}

When an oscillating magnetic field was applied a periodic response was clearly observed in the 2D scattering patterns at low frequencies. For the time channels corresponding to $\mathrm{B}_{\max }= \pm 20 \mathrm{mT}$ the scattering is strongly anisotropic as shown in Fig. 12(a) for $v_{\mathrm{s}}=100 \mathrm{~Hz}$ while for $\mathrm{B}=0$ the patterns are almost isotropic. The SANS intensities averaged over a width of $20^{\circ}$ in sectors at an angle $\alpha=30^{\circ}$ and $90^{\circ}$ are plotted in Fig. $12 \mathrm{~b}$ as a function of Q together with the corresponding curves for the static case. In the static case at $20 \mathrm{mT}$, six-fold symmetry of the pattern is already visible by the peaks at $\mathrm{Q}_{1}\left(\alpha=30^{\circ}\right.$ and $\left.90^{\circ}\right)=0.39 \mathrm{~nm}^{-1}$ corresponding to the in-plane reciprocal lattice vectors, while the shoulder observed at $\mathrm{Q}_{3}=0.29 \mathrm{~nm}^{-1}$ corresponds to the inter-plane vector. Segments of chains are spontaneously formed by dipolar interactions between particle moments giving rise to a characteristic $\mathrm{Q}^{-1}$ dependence of the intensities observed in both sectors at low $\mathrm{Q}$. In the static case, these fragments are partly aligned along the external field and randomly distributed at zero field, while in the dynamic experiment the alignment of the magnetic moments and the local hexagonal arrangement of the nanoparticles are only partly established. Intensities have been integrated in angle sectors $\alpha=0^{\circ}$, $30^{\circ}$ and $90^{\circ}$ over the $\mathrm{Q}$ ranges shown in Fig. $12 \mathrm{~b}$ and denoted by $\mathrm{Q}_{\mathrm{A}}, \mathrm{Q}_{\mathrm{B}}$ and $\mathrm{Q}_{\mathrm{C}}$. The time dependence of the intensities $\mathrm{I}\left(\mathrm{Q}_{\mathrm{i}, \alpha}\right)$ is shown in Fig. $12 \mathrm{c}$ for $\nu_{\mathrm{s}}=100 \mathrm{~Hz}$ for $\mathrm{i}=\mathrm{A}, \mathrm{B}, \mathrm{C}$ and $\alpha=0^{\circ}, 30^{\circ}$ and $90^{\circ}$ and in Fig. 13(a) for different frequencies at $\mathrm{Q}_{B}\left(0^{\circ}\right)$ and $\mathrm{Q}_{\mathrm{B}}\left(90^{\circ}\right)$, respectively. The periodicity of the response is twice that of the magnetic field and the peak positions agree perfectly with the values expected from eq. (14). With increasing frequencies the intensity oscillations decrease in amplitude and fade away above about $v_{\mathrm{s}}=600 \mathrm{~Hz}$. While the intensity levels in Fig. 12c are nearly identical for both time channels $\mathrm{n}_{t}(\mathrm{~B}=0)=35.8$ and 85.8 corresponding to $\mathrm{B}=0$, the amplitudes at $\mathrm{B}=20 \mathrm{mT}$ $\left(n_{t}=11\right)$ are slightly higher than at $B=-20 m T\left(n_{t}=61\right)$. This asymmetry results from a residual static (guide) field of 1-2 mT oriented downwards which superimposes on the applied oscillating field. This effect is quantitatively taken into account in the treatment below. Excellent agreement of all data was obtained by the model function of eq. (14) as shown by the solid lines in Fig. 12c and Fig. 13c when a unique set of parameters was used for all frequencies. In particular, the decrease of the amplitudes and the increase of the apparent gap between the intensities $\mathrm{I}\left(\mathrm{Q}_{\mathrm{B}} 0^{\circ}\right)$ and $\mathrm{I}\left(\mathrm{Q}_{\mathrm{B}}\left(90^{\circ}\right)\right.$ at $\mathrm{n}_{\mathrm{t}}(\mathrm{B}=0)$ result solely from the wavelength smearing. For the sectors $Q_{C}$, the structure factors $S(Q, \alpha)=1$ corresponding to single particle scattering while $S\left(Q_{A}\right)$ and $S\left(Q_{B}\right)$ differ significantly from unity due to inter-plane and 

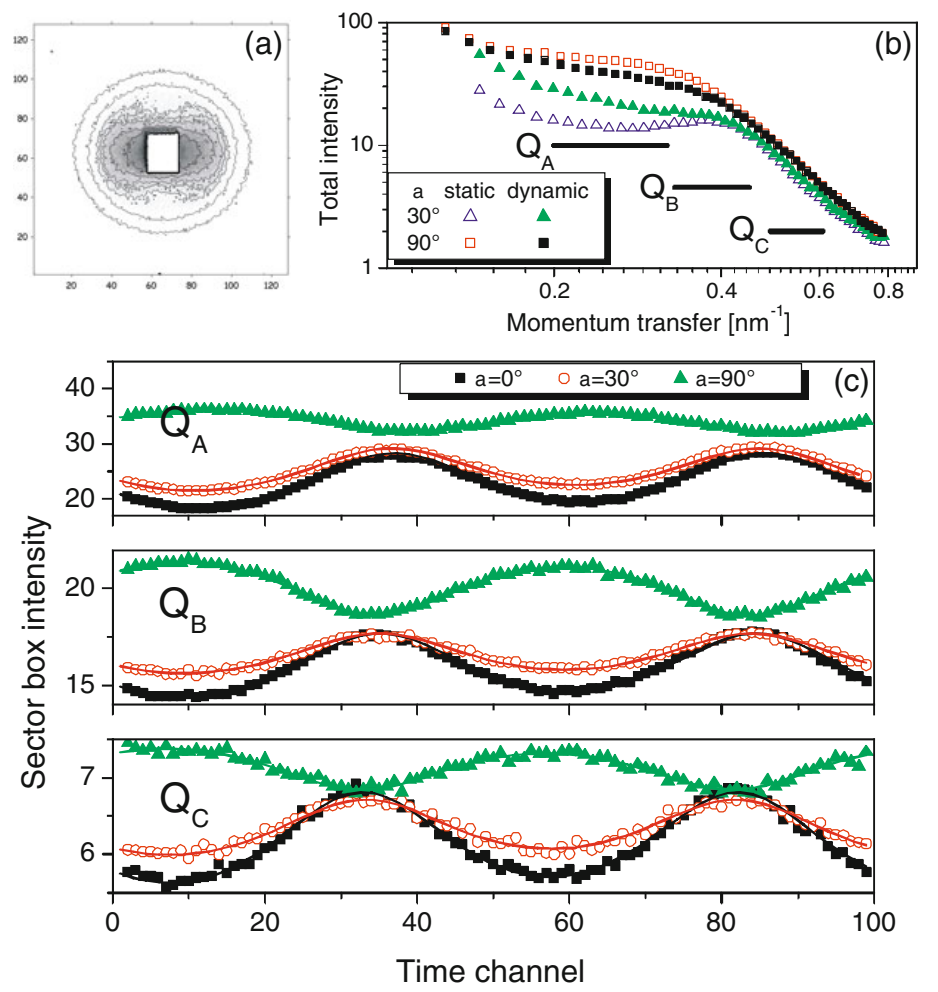

Figure 12. (after [39]). (Color online) SANS intensities (a) in the $2 \mathrm{D}$ pattern and (b) after radial averaging in the static and dynamic $\left(v_{\mathrm{S}}=100 \mathrm{~Hz}, \mathrm{~B}_{\max }= \pm 20 \mathrm{mT}\right)$ modes. (c) Time dependence at $\mathrm{Q}_{\mathrm{A}}, \mathrm{Q}_{\mathrm{B}}$ and $\mathrm{Q}_{\mathrm{C}}$. Solid lines: fits to eq. (14).

intra-chain and in-plane correlations. The intensity plotted in Fig. 13a ranges between $\mathrm{I}\left(\mathrm{Q}_{\mathrm{B}}\left(0^{\circ}\right)\right)=12.4$ and $\mathrm{I}\left(\mathrm{Q}_{\mathrm{B}}\left(90^{\circ}\right)\right)=23.3$, which corresponds to the values as measured in a static magnetic field of $\mathrm{B}=$ $20 \mathrm{mT}$. While the decrease of the amplitudes between 100 and $600 \mathrm{~Hz}$ is explained by the wavelength smearing alone, the amplitude observed at $100 \mathrm{~Hz}$ is only about half of the static case and much lower than the expected value of 0.93 (see above). It clearly indicates that at $100 \mathrm{~Hz}$ only a fraction $\mathrm{f}_{\mathrm{e}}$ of the particle moments are still freely oscillating (eq. (14)). The rest does not follow the external field but remains frozen more or less randomly, leading to the time-independent term $\left(1-\mathrm{f}_{\mathrm{e}}\right) \mathrm{U}\left(\mathrm{Q}, \alpha, v_{\mathrm{s}}\right)$ in eq. (14). In fact, from a simultaneous fit of all data it turned out that $U\left(Q, \alpha, v_{\mathrm{s}}\right)$ is independent of $\alpha$ and $\nu$. It corresponds closely to the intensity as measured in the static mode at $\mathrm{B}=0$ (dashed lines in Fig. 13a) and which is expected for a random orientation of magnetic particle moments in zero magnetic field, where $\mathrm{L}(\mathrm{x}) / \mathrm{x}=1 / 3$ and hence $\mathrm{U}(\mathrm{Q})=\mathrm{F}_{\mathrm{N}}^{2} \mathrm{~S}(\mathrm{Q})+2 / 3 \mathrm{~F}_{\mathrm{M}}^{2}$. The fraction $\mathrm{f}_{\mathrm{e}}$ of superparamagnetic moments that follow the field reversal was found to be of the order of $55 \%$ and nearly constant between $100 \mathrm{~Hz}$ and $600 \mathrm{~Hz}$. At higher frequencies no reliable information on the dynamics can be derived from the continuous stroboscopic technique due to large damping resulting from the wavelength distribution.

\subsection{Response from pulsed stroboscopic technique TISANE}

The 2D scattering patterns in the pulsed TISANE experiment were found to oscillate from nearly isotropic at $n_{\mathrm{t}}(\mathrm{B}=0)$ to strongly anisotropic at $n_{\mathrm{t}}\left( \pm \mathrm{B}_{\max }\right)$. Intensities $\mathrm{I}\left(\mathrm{Q}_{\alpha=0}\right)$ and $\left(\mathrm{Q}_{\alpha=90}\right)$ integrated over full angle sectors of $30^{\circ}$ width, corresponding to an average value of $Q_{B}=0.4 \mathrm{~nm}^{-1}$ are presented in Fig. 13b for several frequencies and different field amplitudes. This shows impressively how the 

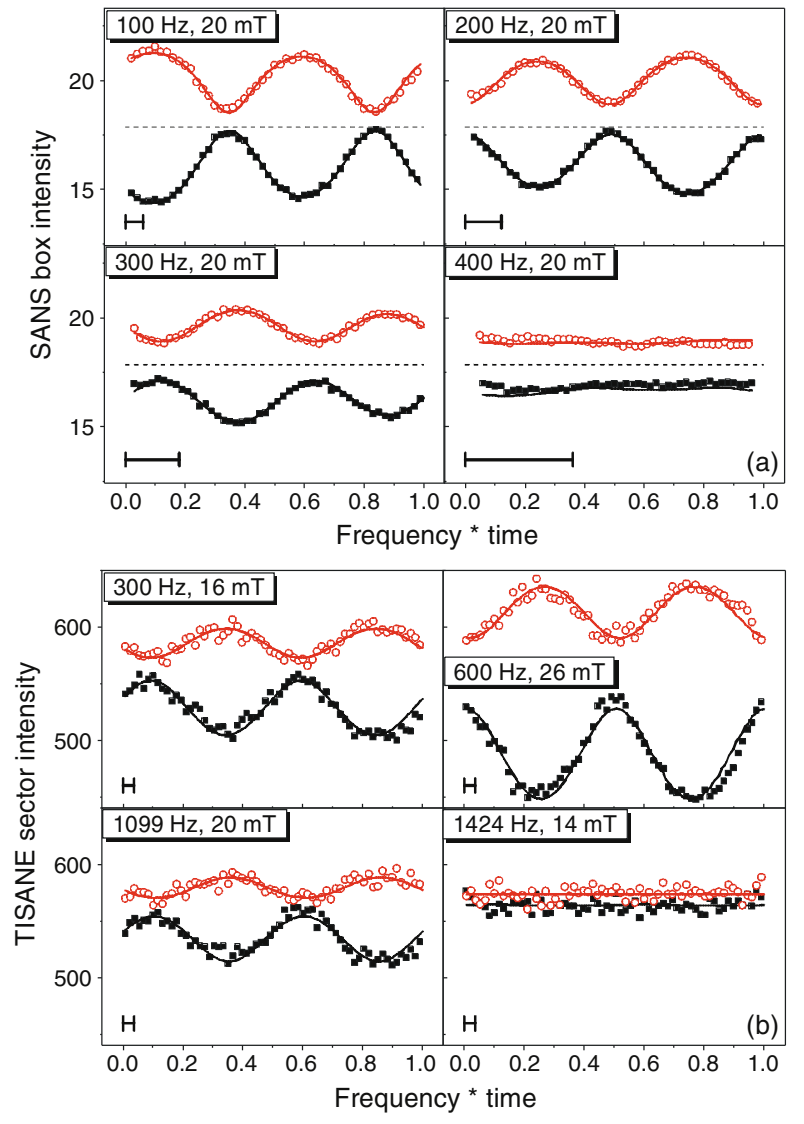

Figure 13. (after [39]). (Color online) Frequency dependence of scattering intensities. Bars indicate the experimental resolution. (a) Stroboscopic SANS mode at $\mathrm{Q}_{B}$. (b) TISANE mode. Symbols: $0^{\circ}$ (closed), $90^{\circ}$ (open). Solid lines: fits to eq. (14). Dashed lines: static SANS mode B $=0$.

pulsed TISANE technique largely extends the frequency range of the continuous mode. Up to about $1300 \mathrm{~Hz}$, the intensities oscillated again with a frequency twice of the B-field while at $1420 \mathrm{~Hz}$ and $2800 \mathrm{~Hz}$ (not shown here) no intensity modulation remained. The frequency dependence of the intensity was analysed as before in terms of eq. (14) using $B_{\mathrm{st}}=0$ and a frequency independent time resolution which corresponds to the actual value of $\Delta \mathrm{t}_{\mathrm{R}} / \mathrm{T}_{\mathrm{s}}=3.5 \%$. The amplitudes of the oscillations measured at $300 \mathrm{~Hz}$ with $\mathrm{B}_{\max }=16 \mathrm{mT}$ and $600 \mathrm{~Hz}$ with $\mathrm{B}_{\max }=26 \mathrm{mT}$, respectively, solely differ due to different values of the Langevin parameters $\mathrm{L}\left(\mathrm{B}_{\max }=16 \mathrm{mT}\right)=0.53$ and $\mathrm{L}\left(\mathrm{B}_{\max }=26 \mathrm{mT}\right)=0.69$, respectively (eq. (4)). The simultaneous fit of the oscillations measured between $300 \mathrm{~Hz}$ and $1099 \mathrm{~Hz}$ at two values of $\mathrm{B}_{\max }$ according to eq. (10) confirmed that the amount of freely oscillating moments $\mathrm{f}_{\mathrm{e}}$ really decreases from $55 \%$ at $300 \mathrm{~Hz}$ and $50 \%$ at $600 \mathrm{~Hz}$ to $30 \%$ at $1099 \mathrm{~Hz}$ and drops to 0 at $1424 \mathrm{~Hz}$. It has to be emphasized that the time independent $2 \mathrm{D}$ pattern at $1424 \mathrm{~Hz}$ was fully isotropic and no gap appeared between $I\left(Q_{\alpha=0}\right)$ and $I\left(Q_{\alpha=90}\right)$ showing the random moment orientation in the fully frozen state. The difference to SANS pattern at $600 \mathrm{~Hz}$ (Fig. 13(a)) is obvious, where the apparent anisotropic behaviour resulted from time averaging of oscillating patterns.

\subsection{Temperature and frequency dependence}

Ferrofluid samples have been cooled down from $300 \mathrm{~K}$ to $100 \mathrm{~K}$ either in zero magnetic field (ZFC) or by applying a static field $B_{\text {st }}$ of $20 \mathrm{mT}$ (FC) [42]. Starting from $100 \mathrm{~K}$, SANS measurements have been 
performed at each temperature in a static field of $\mathrm{B}_{\mathrm{st}} 20 \mathrm{mT}$ and in an oscillating field at frequencies of $100 \mathrm{~Hz}-300 \mathrm{~Hz}$ where the amplitude of the AC field was $\mathrm{B}_{0} \pm 20 \mathrm{mT}$. Basically, all data could be well modelled by eq. (14) by using common structural parameters. The only adjusted parameters were the fraction $f_{e}$ of mobile moments and the structure factors $\mathrm{S}(\mathrm{Q}, \alpha, \mathrm{t})$ which depend on temperature, frequency and on the magnitude field B. Best agreement of the observed intensities was obtained for all angles $\alpha$ with the model function for time-dependent the dynamical structure factors $S(Q, \alpha, t)$ given by

$$
\mathrm{S}(\mathrm{Q}, \alpha, \mathrm{t})=1+(\mathrm{S}(\mathrm{Q}, \alpha)-1) \mathrm{L}^{2}(\mathrm{x})
$$

where $\mathrm{S}(\mathrm{Q}, \alpha)$ gives the time-averaged static structure factor resulting from correlations between neighbouring particles. At zero field, particle moments are randomly oriented and therefore the dynamical structure factor is equal to $S(Q, \alpha, t)=1$. However, as soon as the magnetic field is applied the dynamical structure factor can be larger (for $\alpha=0^{\circ}, 30^{\circ}$ and $90^{\circ}$ ) or smaller (for $\alpha=60^{\circ}$ ) than 1 depending on the actual value of $S(Q, \alpha)$. The formation of field-induced structures is mainly governed by the dipole-dipole coupling which scales with $\mu^{2}$. Therefore, the dynamical structure factor $\mathrm{S}(\mathrm{Q}, \alpha$, t) depends on $\mathrm{L}^{2}(\mathrm{x})$ in this model. The experimental data have been simultaneously fitted with the constraints of common structural parameters (see Table 1 of ref [42]). As a result, the values for the dynamical structure factors $S(Q, \alpha, t)$ can be determined for each temperature. Excellent agreement was obtained when using an identical set of global parameters for both the dynamic data and the static data. The temperature evolution of the model parameters is shown in Fig. 14 for $v=100 \mathrm{~Hz}$ and $\mathrm{B}_{0}=20 \mathrm{mT}$. At $100 \mathrm{~K}$ the static contributions $(1-\mathrm{f}) \mathrm{U}(\alpha, v)$ for $\alpha=0^{\circ}, 30^{\circ}$ and $90^{\circ}$ are nearly identical (Fig. 14) and correspond to those observed in zero field at $250 \mathrm{~K}$. In the frozen state the oscillating part could not be analysed by using the value of a particle moment corresponding to the average volume of the Co core. Instead, the argument $x$ in eq. (4) was adjusted as a free parameter leading to a magnetic particle moment by one order of magnitude smaller. This indicates that at $100 \mathrm{~K}$ only particles with an average core radius of $2.4 \mathrm{~nm}$ can still follow the oscillating magnetic field. Between 100 and $150 \mathrm{~K}$ the static contributions $(1-\mathrm{f}) \mathrm{U}(\alpha, \nu)$ depend more and more on the angle $\alpha$. The dominance of the contribution at $90^{\circ}$ is characteristic for a preferred orientation of chain segments along the field. The temperature dependency of $S(Q, \alpha)$ again demonstrates the dynamical nature of the local ordering: the value of $S(Q$, $\alpha=0^{\circ}$ ) for the direction parallel to $\mathrm{B}_{0}$ is very large at $150 \mathrm{~K}$ and decreases with increasing temperatures while $\mathrm{S}\left(\mathrm{Q}, \alpha=30^{\circ}\right)$ and $\mathrm{S}\left(\mathrm{Q}, \alpha=90^{\circ}\right)$ remain near unity. This indicates that above $\mathrm{T}_{\mathrm{f}}$ an increasing amount of particles are involved in inter-particle correlations forming segments of chains rather than hexagonal layers. The fraction of freely oscillating particle moments $\mathrm{f}_{\mathrm{e}}$ continuously increases from about $20 \%$ at $100 \mathrm{~K}$ (at $100 \mathrm{~Hz}$ ) to $40 \%$ at $300 \mathrm{~K}$ while the static contribution $\mathrm{U}\left(\alpha=90^{\circ}\right)$ decreases.

\subsection{Relaxation mechanisms}

In magnetic colloids, the magnetic field oscillations induce scattering-intensity fluctuations. Once the oscillating field is switched on, larger structures grow that become (partially) aligned when the field reaches its maximum value. Subsequently, the structures either completely disintegrate when the field direction changes orientation, or - if the frequency is high enough - the magnetic moments will change orientation and the particle positions will remain practically unchanged. The formation of these locally ordered domains is mainly governed by magnetic interactions between (single) dipolar chains, while the disintegration of these structures upon reducing the field is predominantly determined by diffusion. For instance, this diffusion-dominated disintegration of dipolar structures is the main reason for hysteresis effects in magnetization curves.

The maximum scattering intensity in the dynamic experiments does not reach the intensity obtained in static fields. This implies that on the time scale of the oscillations in the dynamic experiment $(<10 \mathrm{~ms})$ not all magnetic units become aligned. As it is known from dynamic magnetic susceptibility experiments, the relaxation time strongly depends on the average cluster size. Therefore, it is mainly the presence of aggregates that prevents the scattering intensity from reaching full saturation in the 
a)

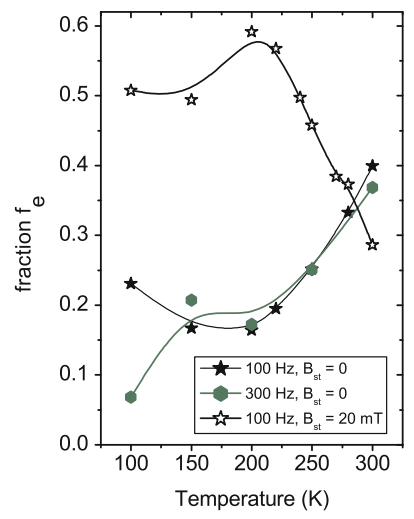

c)

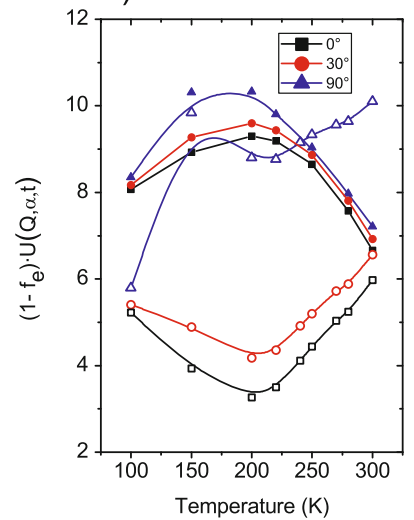

b)

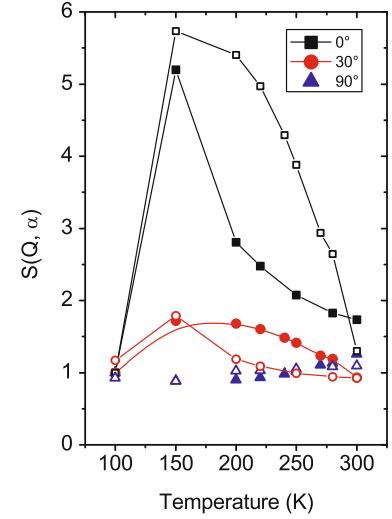

d)

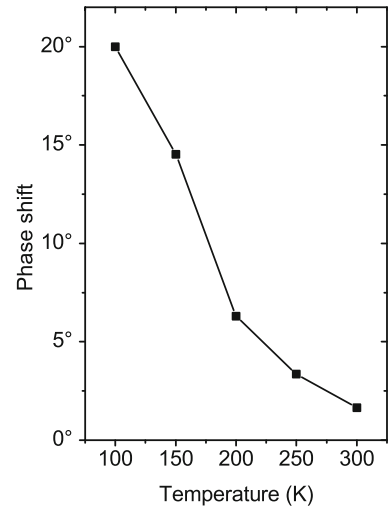

Figure 14. After [42] (Color online). Temperature variation of the model parameters in Co-FF for ZFC data at $100 \mathrm{~Hz}$ and $\mathrm{B} 0=20 \mathrm{mT}$ at $\mathrm{Bst}=0$ (full symbols) and $\mathrm{Bst}=20 \mathrm{mT}$ (open symbols) a) Volume fraction $\mathrm{f}_{\mathrm{e}}$ of oscillating moments. b) Field-induced structure factor $\mathrm{S}\left(\mathrm{Q}, \alpha=0^{\circ}, 30^{\circ}, 90^{\circ}\right)$. c) Static contribution. d) Phase shift extrapolated to $v=0$.

oscillating field. The presented dynamic and static data can be quantitatively described when an effective dynamical structure factor is incorporated that depends on the applied magnetic field by scaling with the square of the Langevin factor. This is unambiguously shown in the $\mathrm{Fe}_{3} \mathrm{O}_{4}$-FF where above the melting point of the ferrofluid, the sector intensities in the direction parallel to the magnetic field oscillate in phase with the sector intensities perpendicular to the field. If the structure factor would have no effect on the applied field then the scattering intensity in the $0^{\circ}$ direction would always be maximum in zero field.

Thermal reorientation of the magnetic moment in a magnetic colloid can take place either by Brownian rotation of the particle or/and by Néel relaxation of the particle moment. The first mechanism has a relaxation time $\tau_{B}$ that depends on the hydrodynamic radius $\mathrm{R}_{\mathrm{h}}$ and the viscosity $\eta$ of the solvent according to:

$$
\tau_{B}=\frac{4 \cdot \pi \cdot \eta \cdot R_{h}^{3}}{k_{B} \cdot T}
$$

where $k_{B}$ is the Boltzmann constant and $\mathrm{T}$ the absolute temperature. The Néel mechanism involves rotation of the magnetization vector inside a particle against an energy barrier $\mathrm{K}_{\mathrm{a}} \mathrm{V}_{\mathrm{c}}$ with a characteristic 


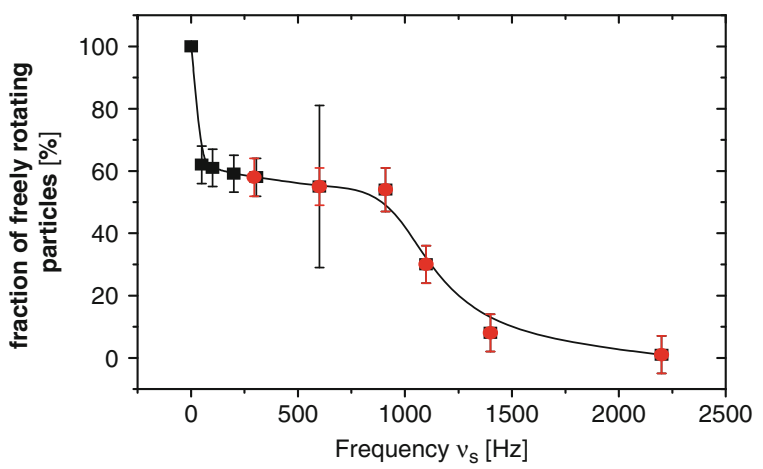

Figure 15. Fraction of freely rotating particle moments as a function of frequency of oscillating magnetic field.

time $\tau_{\mathrm{N}}$ given by:

$$
\tau_{N}=\frac{1}{2 \cdot \pi \cdot f_{0}} \cdot \exp \left(\frac{K_{a} \cdot V_{c}}{k_{B} \cdot T}\right)
$$

$f_{0}$ is a constant of the order of $10^{9} \mathrm{~s}^{-1}, \mathrm{~K}_{\mathrm{a}}$ is a shape-dependent material constant and $\mathrm{V}_{\mathrm{c}}$ is the volume of the magnetic core for a sphere with radius $R_{c}$. The apparent characteristic time is given by $\tau(\mathrm{app})^{-1}=\tau_{\mathrm{B}}^{-1}+\tau_{\mathrm{N}}$ which means that the fastest mechanism dominates for individual particles.

Dipolar interactions between particles influence both the Néel and the Brownian mechanisms either by formation of larger domains with correspondingly longer relaxation times $\tau_{\mathrm{B}}$ or by modification of the energy barrier $\mathrm{K}_{\mathrm{a}} \mathrm{V}_{\mathrm{c}}$ in eq. (15) longer [47] or shorter relaxation times have been predicted [48] and indications of collective phenomena have been reported [49]. Using the structural parameters of the sample "MFT3" $\left(\mathrm{R}_{\mathrm{c}}=4.4 \pm 02 \mathrm{~nm}\right)$ and $\mathrm{K}=2.6105 \mathrm{~J} \cdot \mathrm{m}^{-3}$ for fcc-Co, Néel relaxation times are expected of the order of $100 \mathrm{~s}$, i.e. at room temperatures almost all the magnetic moments are blocked inside the Co particles. The Brownian relaxation time $\tau_{\mathrm{B}}$ expected for single core-shell particles of radius $\mathrm{R}_{\mathrm{p}}=6.4-9.5 \mathrm{~nm}$ and the viscosity $\eta=0.2$ Pa.s for $\mathrm{L} 9$, ranges between 0.16 and $0.5 \mathrm{~ms}$, which is by four orders of magnitude faster than observed. Reversely, Brownian relaxation times of $2-5 \mathrm{~s}$ as observed when the system relaxes towards equilibrium would imply large aggregates of the order of $150-200 \mathrm{~nm}$ which effectively corresponds to the size of domains which has been detected from the width of the static correlation peaks [25].

The data combined from the continuous and TISANE techniques indicate a continuous relaxation process (Fig. 15), which is basically in agreement with AC susceptibility measurements [42]. For about $40 \%$ of the particles, moment rotation is blocked already below $100 \mathrm{~Hz}$ while between 600 and $1300 \mathrm{~Hz}$ the remaining particle moments continuously freeze out in a random orientation. The step around 1000$1300 \mathrm{~Hz}$ corresponds closely to the characteristic time $\tau_{\mathrm{B}}=160 \mu$ s expected for Brownian rotational diffusion of individual core-shell particles of radius $R_{\mathrm{p}}=\mathrm{R}_{\mathrm{c}}+\mathrm{D}=6.4 \mathrm{~nm}$ and a viscosity $\eta=0.2$ Pa.s for the liquid "L9", according to eq. (15). The field-induced ordering process must be governed by the fast particle rotation causing the magnetic moments to align along the direction of the magnetic field. Then the moments get stuck into locally ordered domains of about $100 \mathrm{~nm}$ in size. Magnetic relaxation takes place either by Brownian rotation of the whole domain or by rotation of magnetic moment inside the particle against the anisotropy energy barrier according to the Néel mechanism given by eq. (17). The characteristic time of both mechanisms is expected to be $1-50 \mathrm{~s}$, which corresponds closely to the values observed previously when the field was switched off allowing for full relaxation into equilibrium [44]. This slow relaxation of large ordered domains is at the origin of the decrease of $f$ in Fig. 15 at 
low frequencies. The incomplete relaxation in an oscillating magnetic field gives rise to frozen random orientation of particle moments observed as time independent scattering contribution.

When the ferrofluids are cooled down below the melting point of the carrier liquid the dynamics is almost arrested. Brownian rotation of the individual particles or structures is prevented and relaxation of the magnetic moments according to the Néel mechanism inside the particle cannot take place due to the anisotropy energy $\mathrm{K}_{\mathrm{a}} \mathrm{V}_{\mathrm{c}} \geq \mathrm{k}_{\mathrm{B}} \mathrm{T}$. The effect of the temperature on the dynamical structure factor can be described quantitatively using the presented model. At temperatures far below the melting point of the solvent, the structure factor is close to unity, manifesting only relatively small structures if cooling is performed in zero field. Upon increasing the temperature the dynamical structure factor increases and reaches a maximum just above the melting point in the direction perpendicular to the field. Subsequently, when the temperature is increased to room temperature the structure factor decreases again as a result of growing thermal fluctuations. The temperature dependency of the structure factor indicates that the formation of dipolar structures is mainly determined by the effective dipole-dipole interaction, which is enhanced by the (partial) alignment of the dipoles when an external magnetic field is applied. In the case of $\mathrm{Fe}_{3} \mathrm{O}_{4}$-FF the dominating structure factor is $\mathrm{S}\left(\mathrm{Q}, \alpha=90^{\circ}\right)$ which reflects the formation of hexagons while for Co-FF at the same strength of magnetic field $\mathrm{S}\left(\mathrm{Q}, \alpha=0^{\circ}\right)$ is dominating which reflects the presence of chain-like correlations. Furthermore, the number of particles that can follow the oscillations drops significantly with decreasing temperature due to the formation of larger structures with longer relaxation times and it tends to zero below $\mathrm{T}_{\mathrm{f}}$.

Clearly, "field-induced ordered domains" in ferrofluids cannot be considered as static units. Due to the fast rotation of individual particle moments and slow relaxation of larger domains the system would end up fully arrested after a large number of oscillations. Our measurements showed instead that the process above $T_{\mathrm{f}}$ is fully reversible and that there is no difference between the corresponding intensities measured at the beginning and at the end of the experiment. Instead, the domains have to be considered as "living objects": Particles can move away from one domain by translational diffusion in the liquid and will be for some time free where in an ultimate oscillation cycle they will be stuck to a different domain. At a given temperature the local ordering is characterised by the dynamical structure factors and the size of domains represents a time averaged value corresponding to dynamical equilibrium. Below the freezing temperatures the dynamics is almost fully arrested.

\section{CONCLUSION}

Complementary to conventional Small Angle Neutron Scattering, polarised neutrons are a powerful tool in the investigation of spatial magnetisation and density fluctuations at surface and interfaces of nanoscaled structures. In SANSPOL, the relative contrasts are drastically modified which allows any chemical or magnetisation gradients to be established more precisely. In multi-phase systems this technique allows magnetic and non-magnetic inhomogeneities to be distinguished even when they are of similar sizes. The magnetisation behaviour of small particles can be precisely determined from the nuclear-magnetic cross-term even at low magnetic fields, where the moments are only partially aligned. SANSPOL allows further to separate the scattering resulting from particle form factors alone from the appearance of correlation peaks which reflect the onset of inter-particle interactions. In addition, polarised neutrons gives the relative sign of nuclear and magnetic contrasts and therefore monitors depletion zones and magnetically inactive layers at surfaces or interfaces. Stroboscopic SANS technique allows the dynamics of magnetic nanoparticles' moments to be studied on a sub-millisecond time scale: This is well above the limitations of Neutron Spin-Echo techniques [50] or Mössbauer spectroscopy [51] $\left(10^{-6}-10^{-12} \mathrm{~s}\right)$ and is complementary to X-ray Photon Correlation Spectroscopy [52] and Forced Rayleigh Scattering [53]. The stroboscopic SANS technique allowed elucidation of the dynamical nature of the locally ordered domains in both ferrofluids as "living objects" getting arrested below the freezing of the solvent. 


\section{Acknowledgements}

The author is very grateful to the long term collaboration with U. Keiderling, S. Prévost, A. Hoell, M. Kammel, K. Habicht and M. Russina and from Helmholtz-Zentrum, Berlin, R. Gähler, Ch. Dewhurst and R.P. May from the Institut Laue Langevin, Grenoble, B. Erné and M. Klockkenburg from the University of Utrecht and J. Kohlbrecher from PSI, Wuerenlingen. This research has been supported by the European Commission under the 6th Framework through the Key Action: Strengthening the European Research Infrastructures. Contract n: RII-CT-2003-505925 and by the German Research Foundation DFG Project No Wi-1151/3.

\section{References}

[1] A. Wiedenmann, J. Appl. Cryst., 30 (1997) 580-585.

[2] J. F. Loeffler, H. B. Braun, W. Wagner, G. Kostorz, A. Wiedenmann Materials Science and Engineering A 304-306, 31 (2001) 1050-1054.

[3] J. Weissmüller, A. Michels, D. Michels, A. Wiedenmann, C.E. Krill, H.M. Sauer, R. Birringer Phys. Rev. B (2004) vol. 69, no. 5, p. 54402-1-16.

[4] G. Kostorz in Treatease on Materials Science and Technology, Acad. Press (1979) ed. G. Kostorz, 227-290.

[5] J.K. Percus, G.J. Yevick Phys. Rev. 110 (1959) 1-13.

[6] J.S. Pedersen J. Appl. Cryst. 27 (1994) 595-608.

[7] R.M. Moon, T. Riste, \& W.C. Koehler, Phys. Rev. 181 (1969) 920-931.

[8] R. Pynn, \& J. B.Hayter. Phys. Rev. Letters, 51 (1983) 710-713.

[9] A. Wiedenmann, Mat. Science Forum, 312-314 (1999) 315.

J. Metastable and Nanocrystalline Materials, 2-6 (1999) 315.

[10] A. Wiedenmann, J. Appl. Cryst. 33 (2000) 428-432.

[11] A. Wiedenmann, Physica B 297 (2001) 226-233.

[12] J. Kohlbrecher, A. Wiedenmann, H. Wollenberger, Z. Physik, 104 (1997) 1-4.

[13] J. Kohlbrecher, PhD Thesis Technische Universität Berlin 1996.

[14] A. Heinemann, A. Wiedenmann J. Appl. Cryst. 36 (2003) 845-849.

[15] U. Keiderling \& A. Wiedenmann, A. Physica B, $213 \& 214$ (1995) 895-897.

[16] T. Keller, T. Krist, A. Danzig, U. Keiderling, F. Mezei, A. Wiedenmann, J. Nuclear Instruments A 451 (2000) 474-479.

[17] A. Wiedenmann in Magnetically Controllable Fluids and Their Applications, edited by S. Odenbach, Lecture Notes in Physics, Vol. 594, Springer, Berlin, (2002) 33-58.

[18] N. Buske, DE patent 19758350 (1997).

[19] A. Heinemann, A. Wiedenmann JMMM 289 (2005) 149-151.

[20] M. Kammel, A. Hoell, A. Wiedenmann, Scripta Materialia 44 (2001) 2341-2345.

[21] M. Kammel, A. Wiedenmann, A. Hoell, J. Magn. Magn. Mater. 252 (2002) 89-91.

[22] A. Hoell, M. Kammel, A. Heienmann, A. Wiedenmann J. Appl. Cryst. 36 (2003) 558-561.

[23] K. Butter, P. Bomans, P. Frederik, G. Vroege, and A. Philipse, Nature Materials 2 (2003) 88-91.

[24] K. Butter, A. Wiedenmann, A. Hoell, A. Petukhov, G.J. Vroege, J. Appl. Cryst. 37 (2004) 847-856.

[25] A. Wiedenmann, A. Hoell, M. Kammel, P. Boesecke, Phys. Rev E 68 (2003) 031203.

[26] A. Wiedenmann, A. Heinemann JMMM 289 (2005) 58-61.

[27] P.G. de Gennes, P.A. Pincus, Phys. Condens. Mater. 11 (1970) 189.

[28] J. Liu, E. Lawrence, A. Wu, M. Ivey, G. Flores, K. Javier, J. Bibette, and J. Richards, Phys. Rev. Lett. 74 (1995) 2828-3831.

[29] A. Hoell, A. Wiedenmann, U. Heyen, D. Schüler Physica B 350 (2004) e309-e313.

[30] S. Hess, J.B. Hayter, R. Pynn Mol. Phys 53 (1984) 1527.

[31] B. Groh and S. Dietrich, Phys. Rev. E 63 (2001) 021203.

[32] J.B. Hayter. J. Appl. Cryst., 21 (1988), 737-742 
[33] S. Hess, edited by S. A. Safran and N. A. Clark _Wiley Interscience, New York, 1987_, pp. 631-642.

[34] U. Lembke, A. Hoell, R. Kranold, R. Müller, G. Goerick, R. Gilles, A. Wiedenmann, J. Appl. Physics, 85 (1999) 2279-2286.

[35] A. Wiedenmann, U. Lembke, A. Hoell, R. Müller, W. Schüppel. Nanostructured materials, 12 (1999) 601-604.

[36] Y. Yoshizawa, S. Oguma, K.J. Yamauchi, Appl. Phys, vol. 64 (1988) 6044-6064.

[37] J. Kohlbrecher, A. Wiedenmann, H. Wollenberger, Mater. Sci. Forum, vol. 225-227 (1996) 677.

[38] J. Kohlbrecher, A. Wiedenmann, Physica B241-243 (1998) 591-593.

[39] A. Wiedenmann, U. Keiderling, K. Habicht, M. Russina and R. Gähler, Phys. Rev. Lett. 97 (2006) 057202.

[40] R. Gähler and R. Golub, Z. Phys. B 56 (1984) 5.

[41] D. Kippling, R. Gaehler, K. Habicht Physics Lett A 372 (2008) 154-1547.

[42] A. Wiedenmann, U. Keiderling, M. Meissner, D. Wallacher, R. Gähler, R.P. May, S. Prévost, M. Klokkenburg, B. Erné, J. Kohlbrecher, Phys. Rev. B77 (2008) 184417.

[43] M. Klokkenburg, B. H. Erné, J. D. Meeldijk, A. Wiedenmann, A. V. Petukhov, R. P. A. Dullens, and A. P. Philipse, Phys. Rev. Lett. 97 (2006) 185702.

[44] A. Wiedenmann, U. Keiderling, R. May and C. Dewhurst, Physica B 385 (2006) 453-456.

[45] M. Klokkenburg, B. H. Erné, A. Wiedenmann, A. V. Petukhov, and A. P. Philipse, Phys. Rev. E 75 (2007) 051408.

[46] A. Wiedenmann, Physica B 356 (2005) 246-253.

[47] F. Luis, F. Petroff, J.M. Torres, L.M. Garcia, J. Bartolomé, J. Carrey and A. Vaurès, Phys. Rev. Lett. 88 (2002) 217205.

[48] S. Morup and E. Tronc, Phys. Rev. Lett. 72 (1994) 3278.

[49] C. Djurberg, P. Svedlindh, P. Nordblad, M. F. Hansen, F. Bodker and S. Morup, Phys. Rev. Lett. 79 (1997) 5154.

[50] H. Casalta, P. Schleger, C. Bellouard, M. Hennion, I. Mirebeau, G. Ehlers, B. Farago, J. L Dormann, M. Kelsch, M. Linde and F. Phillipp, Phys. Rev. Lett. 82 (1999) 1301.

[51] M. F. Hansen, F. Bodker, S. Morup, K. Lefmann, K. N. Clausen and P. A. Lindgard, Phys. Rev. Lett. 79 (1997) 4910.

[52] J. Lal, D. Abernathy, L. Auvray, O. Diat and G. Grübel, Eur. Phys. E 4 (2001) 263.

[53] J. C. Bacri, A. Cebers, A. Bourdon, G. Demouchy, B. M. Heegaard and R. Perzynski, Phys. Rev. Lett. 74 (1995) 5032. 